\title{
A study of the Keplerian accretion disk and precessing outflow in the massive protostar IRAS 20126+4104
}

\author{
R. Cesaroni ${ }^{1}$, R. Neri ${ }^{2}$, L. Olmi ${ }^{3}$, L. Testi ${ }^{1}$, C. M. Walmsley ${ }^{1}$, and P. Hofner ${ }^{4,5}$ \\ 1 Osservatorio Astrofisico di Arcetri, INAF, Largo E. Fermi 5, 50125 Firenze, Italy \\ e-mail: cesa@arcetri.astro.it \\ 2 IRAM, 300 rue de la Piscine, Domaine Universitaire, 38406 St. Martin d'Hères Cedex, France \\ 3 Istituto di Radioastronomia, CNR, Sezione di Firenze, Largo E. Fermi 5, 50125 Firenze, Italy \\ ${ }^{4}$ Physics Department, New Mexico Tech, 801 Leroy Place, Socorro, NM 87801, USA \\ 5 National Radio Astronomy Observatory, PO Box O, Socorro, NM 87801, USA
}

Received 12 July 2004 / Accepted 16 January 2005

\begin{abstract}
We report on interferometric observations at 3.2 and $1.3 \mathrm{~mm}$ of the massive young stellar object IRAS 20126+4104 obtained in the $\mathrm{C}^{34} \mathrm{~S}$ and $\mathrm{CH}_{3} \mathrm{OH}$ lines and in the continuum emission. The $\mathrm{C}^{34} \mathrm{~S}$ data confirm the existence of a Keplerian disk, as already suggested by various authors. However, the mass of the central object is $\sim 7 M_{\odot}$, significantly less than previous estimates. We believe that such a discrepancy is due to the fact that the rotation curve is affected not only by the star but also by the mass in the innermost regions of the disk itself: this leads to an overestimate of the stellar mass when low-density tracers are used to study the velocity field over regions larger than a few seconds of arc (i.e. a few $0.01 \mathrm{pc}$ ). On the basis of the line profiles we speculate that accretion onto the star might be still occurring through the disk. This seems consistent with current models of high-mass star formation which predict an accretion luminosity equal to that of IRAS $20126+4104$ for a $7 M_{\odot}$ protostar. The

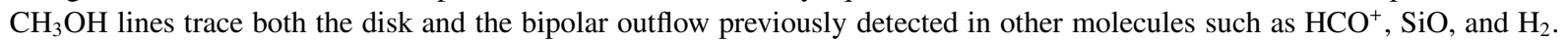
New $\mathrm{H}_{2}$ images obtained at $2.2 \mu \mathrm{m}$ confirm that the outflow axis is undergoing precession. We elaborate a simple model that suitably fits the data thus allowing derivation of a few basic parameters of the precession.
\end{abstract}

Key words. stars: formation - ISM: jets and outflows - ISM: individual objects: IRAS 20126+4104

\section{Introduction}

Both theory and observations suggest a model for star formation which proceeds through inside-out collapse of a molecular core and accretion onto a protostellar nucleus. The fate of such a protostar however depends on the amount of material available for accretion. If this reservoir is quickly exhausted, then the protostar slowly contracts until nuclear reactions switch on and the star reaches the zero-age main sequence (ZAMS). Otherwise accretion goes on and the protostar grows till the critical mass is reached beyond which its contraction becomes faster than accretion: this should happen at about $8 M_{\odot}$ (Palla \& Stahler 1993). At this point, the protostar collapses and ignites hydrogen burning reaching the ZAMS: the luminosity thus generated prevents further accretion due to the powerful radiation pressure exerted on the surrounding material. This scenario has the consequence that massive (i.e. earlytype) stars should not form through accretion. Such a conclusion is the consequence of the simplistic treatment of the star formation process outlined above. Accretion rates sufficiently

^ Based on observations carried out with the IRAM Plateau de Bure Interferometer. IRAM is supported by INSU/CNRS (France), MPG (Germany) and IGN (Spain). large $\left(\geq 10^{-3} M_{\odot} \mathrm{yr}^{-1}\right)$ to overcome radiation pressure cannot be excluded. Moreover, magnetic fields and angular momentum conservation likely cause flattening of the collapsing core and hence formation of circumstellar accretion disks which are much less affected by radiation pressure. As a matter of fact, such disks have been found in T Tauri stars (see e.g. Simon et al. 2001), but it is of crucial importance to prove their existence also in association with high-mass stars.

With this in mind, we conducted a detailed study of a luminous $\left(\sim 10^{4} L_{\odot}\right)$ young stellar object (YSO) located at a relatively small distance $(1.7 \mathrm{kpc})$ : IRAS $20126+4104$. This object was first identified in the IRAS point source catalogue on the basis of its far infrared colours typical of ultracompact HII regions (according to the classification by Wood \& Churchwell 1989) and of its association with water maser emission (Comoretto et al. 1990). Tofani et al. (1995) performed VLA observations at 1.3 and $3.6 \mathrm{~cm}$ towards this source: no continuum emission was detected but it could be established that the $\mathrm{H}_{2} \mathrm{O}$ maser spots lie along a SE-NW direction, with velocity increasing from NW to SE. Subsequent single-dish observations by Cesaroni et al. (1999b) demonstrated that the IRAS source and associated $\mathrm{H}_{2} \mathrm{O}$ masers are located inside a dense, parsec-scale molecular clump, as already suggested by 
Table 1. Frequency set-up for the PdBI observations. SSB tuning was used at $3.2 \mathrm{~mm}$ and DSB at $1.3 \mathrm{~mm}$.

\begin{tabular}{|c|c|c|c|c|}
\hline \multirow[t]{2}{*}{ Line } & \multirow{2}{*}{$\begin{array}{c}\text { Centre frequency } \\
(\mathrm{MHz})\end{array}$} & \multirow{2}{*}{$\begin{array}{l}\text { Bandwidth } \\
\text { (MHz) }\end{array}$} & \multicolumn{2}{|c|}{ Channel spacing } \\
\hline & & & $(\mathrm{MHz})$ & $\left(\mathrm{km} \mathrm{s}^{-1}\right)$ \\
\hline $\mathrm{C}^{34} \mathrm{~S}(2-1)$ and $\mathrm{CH}_{3} \mathrm{OH}(2-1) v_{\mathrm{t}}=1$ & 96356 & 40 & 0.078125 & 0.243 \\
\hline $\mathrm{C}^{34} \mathrm{~S}(2-1)$ and $\mathrm{CH}_{3} \mathrm{OH}(2-1) v_{\mathrm{t}}=1$ & 96356 & 320 & 2.5 & 7.778 \\
\hline $\mathrm{CH}_{3} \mathrm{OH}(2-1) v=0$ & 96749 & 40 & 0.078125 & 0.242 \\
\hline $\mathrm{CH}_{3} \mathrm{OH}(2-1) v=0$ & 96551 & 160 & 1.25 & 3.881 \\
\hline $\mathrm{CH}_{3} \mathrm{OH}(2-1) v_{\mathrm{t}}=1$ & 96691 & 160 & 1.25 & 3.876 \\
\hline$C^{34} S(5-4)$ & 241017 & 40 & 0.078125 & 0.097 \\
\hline $\mathrm{C}^{34} \mathrm{~S}(5-4)$ and $\mathrm{CH}_{3} \mathrm{OH}(5-4) v_{\mathrm{t}}=1$ & 241144 & 320 & 2.5 & 3.108 \\
\hline $\mathrm{C}^{34} \mathrm{~S}(5-4)$ and $\mathrm{CH}_{3} \mathrm{OH}(5-4) v_{\mathrm{t}}=1$ & 240884 & 320 & 2.5 & 3.111 \\
\hline
\end{tabular}

the results of Estalella et al. (1993), and confirmed the existence of a bipolar outflow oriented $\mathrm{N}-\mathrm{S}$, previously detected by Wilking et al. (1990). Follow-up interferometric observations (Cesaroni et al. 1997, hereafter C97; Cesaroni et al. 1999a, hereafter C99) revealed an unexpected complexity, making of IRAS 20126+4104 the most promising candidate of a massive protostar with a disk-jet system. Surprisingly, the outflow structure revealed in the high-angular resolution maps is dramatically different from that seen on a larger scale: not only the orientation is NW-SE instead of N-S, but the velocity of the lobes reverses so that, e.g., the blue-shifted gas is located to the SE at low velocities, whereas it appears to the NW at high velocities. Such a velocity reversal has remained unexplained to date: in Sect. 4.2.2 we propose a solution to this problem. On the other hand, the change of orientation of the flow axis from the large to the small scale has been interpreted by Shepherd et al. (2000; hereafter SYBT) in terms of precession of the outflow. $\mathrm{SiO}$ and $\mathrm{H}_{2}$ images of a bipolar jet powering the flow have been obtained by $\mathrm{C} 97$ and C99 thus allowing an accurate estimate of the jet axis orientation in space and its expansion velocity. Such a jet has been traced down to scales as small as a few $100 \mathrm{AU}$ by means of VLA observations of the $3.6 \mathrm{~cm}$ continuum emission (Hofner et al. 1999) and VLBA observations of the $\mathrm{H}_{2} \mathrm{O}$ maser spots (Moscadelli et al. 2000). Finally, the most striking finding in IRAS 20126+4104 is the detection of a Keplerian circumstellar disk (C97; C99; Zhang et al. 1998) approximately perpendicular to the jet. This result supports the hypothesis that the formation of O-B stars could proceed by accretion through disks, like in the case of low-mass stars.

In view of the results obtained so far, IRAS 20126+4104 turns out to be a unique tool to achieve a deeper understanding of the formation mechanism of massive stars. We thus decided to conduct further high angular resolution observations with the goal of achieving a clear picture of the disk structure and Keplerian velocity field, and hence a precise estimate of the stellar mass. At the same time, we wished to better investigate the morphology and kinematics of the flow and possibly assess the existence of infall, as suggested by C99.

\section{Observations}

\subsection{Plateau de Bure interferometer}

The interferometric observations were carried out between January and March 2002 using the IRAM six element array at Plateau de Bure, France (Guilloteau et al. 1992). The 15-m antennas were equipped with $82-116 \mathrm{GHz}$ and $210-245 \mathrm{GHz}$ SIS receivers operating simultaneously with double-sideband (DSB) receiver temperatures of $35 \mathrm{~K}$ at $3 \mathrm{~mm}$ and $50 \mathrm{~K}$ at $1.3 \mathrm{~mm}$. The receivers were tuned single side-band at $3.2 \mathrm{~mm}$ and double side-band at $1.3 \mathrm{~mm}$. The facility correlator was centred at $96.526 \mathrm{GHz}$ in the upper side-band at $3.2 \mathrm{~mm}$ and at $241.054 \mathrm{GHz}$ in the upper side-band at $1.3 \mathrm{~mm}$. We show the frequency set-up of the correlator and list the detected spectral lines in Table 1. The eight units in the correlator were placed in such a way that a frequency range free of lines could be used to measure the continuum flux. The effective spectral resolution of the correlator is about twice the nominal channel spacings given in Table 1.

Source visibilities were phase calibrated by means of interspersed observations (every $20 \mathrm{~min}$ ) of the nearby point source $2013+370$. The bandpass calibration was carried out in the antenna-based manner. The flux of the primary calibrators was bootstrapped from IRAM monitoring measurements and used to derive the absolute flux density scale. Table 2 gives a list of the main parameters for our PdBI observations.

The calibration and data reduction were made using the standard IRAM/GAG software. Continuum subtraction was performed in the image plane by using a continuum map obtained by averaging all line free channels in the $580 \mathrm{MHz}$ wide lower- and upper-side bands. Finally, channel maps were produced for all the lines. The conversion factor from flux to brightness temperature in the synthesised beam is approximately $\sim 20 \mathrm{~K}(\mathrm{Jy} / \text { beam })^{-1}$ at $3.2 \mathrm{~mm}$ and $\sim 30 \mathrm{~K}(\mathrm{Jy} / \text { beam })^{-1}$ at $1.3 \mathrm{~mm}$ for the synthesised beams given in Table 2 .

\subsection{TNG observations}

IRAS $20126+4104$ was observed in July 2001 using the $3.5-\mathrm{m}$ Telescopio Nazionale Galileo (TNG) equipped with the near infrared camera spectrograph (NICS, Baffa et al. 2001). A region of approximately $4 \times 4$ arcmin centered on the IRAS source was imaged in the $\mathrm{H}_{2}(\lambda=2.12 \mu \mathrm{m})$ narrow band filter and in a nearby narrow-band continuum filter, $K_{\text {cont }}$, a detailed characterization of all these filters can be found in Ghinassi et al. (2002). A series of dithered exposure were obtained in each filter and used to remove the sky emission. The final integration times per sky position was $30 \mathrm{~min}$ in each of the $\mathrm{H}_{2}$ and $K_{\mathrm{c}}$ filters. Data reduction and analysis were performed using the IRAF software package, following standard flat-fielding 
Table 2. Instrumental parameters for the IRAM PdBI observations.

\begin{tabular}{lc}
\hline \hline \multicolumn{1}{c}{ Parameter } & Value \\
\hline Centre position & $\alpha(J 2000)=20^{\mathrm{h}} 14^{\mathrm{m}} 26^{\mathrm{s}} .036$ \\
& $\delta(J 2000)=41^{\circ} 13^{\prime} 32^{\prime \prime} 52$ \\
Number of antennas & 6 \\
Baseline range & $48-331 \mathrm{~m}$ \\
Band centre & 96.526 and $241.054 \mathrm{GHz}$ \\
Total correlator bandwidth & $580 \mathrm{MHz}$ \\
Number of sections in the correlator & 8 \\
Primary HPBW & $50^{\prime \prime}$ at $3.2 \mathrm{~mm}$ and $20^{\prime \prime}$ at $1.3 \mathrm{~mm}$ \\
Synthesised HPBW & $\sim 2^{\prime \prime} 4$ at $3.2 \mathrm{~mm}$ and $\sim 0.85$ at $1.3 \mathrm{~mm}$ \\
Primary flux density calib. & $3 \mathrm{C} 273: 9.7-11.8 \mathrm{Jy}$ at $3.2 \mathrm{~mm} ; 3.7-7.8 \mathrm{Jy} 1.3 \mathrm{~mm}$ \\
& $3 \mathrm{C} 345: 4.7 \mathrm{Jy}$ at $3.2 \mathrm{~mm} ; 4.0 \mathrm{Jy}$ at $1.3 \mathrm{~mm}$ \\
Phase and amplitude calib. & $2013+370: 2.7-3.1 \mathrm{Jy}$ at $3.2 \mathrm{~mm} ; 1.5-3.0 \mathrm{Jy}$ at $1.3 \mathrm{~mm}$ \\
\hline
\end{tabular}

and sky subtraction, the individual images were registered and the final mosaic was produced. No flux calibration was attempted as the night was not photometric. The continuum emission was removed from the $\mathrm{H}_{2}$ image using the $K_{\mathrm{c}}$ image, the procedure works very well in most cases except for the brightest or steep spectrum stellar sources which left a positive or negative residual. Accurate $(\leq 0,5)$ astrometry was derived for both mosaics using stellar positions from the 2MASS second incremental data release. The angular resolution of the observation was limited to $\sim 0$. $^{\prime} 9$ by the seeing conditions.

\section{Results}

Both $\mathrm{C}^{34} \mathrm{~S}$ lines, all ground state transitions of $\mathrm{CH}_{3} \mathrm{OH}(2-1)$, and most of the vibrationally excited lines of $\mathrm{CH}_{3} \mathrm{OH}(2-1)$ and (5-4) covered by our frequency setup have been detected. As for $\mathrm{CH}_{3} \mathrm{CN}$ (see $\mathrm{C} 97$ and $\mathrm{C} 99$ ), also the $\mathrm{C}^{34} \mathrm{~S}$ and $\mathrm{CH}_{3} \mathrm{OH}$ lines arise from a compact molecular core of $\sim 1000 \mathrm{AU}$, also traced by the continuum emission at 3.2 and $1.3 \mathrm{~mm}$. This can be seen in Fig. 1, where maps of the integrated emission in these lines are superposed on the corresponding continuum emission. It is worth noting that only $\leq 1 / 3$ of the $C^{34} S$ emission measured with the 30-m telescope by Cesaroni et al. (1999b) is imaged in the PdBI observations, which indicates that most of the emission originates from a region much larger than the $\lesssim 1^{\prime \prime}$ core.

In Table 3 we list the parameters of the continuum emission. The angular diameters $(\Theta)$ have been obtained with Gaussian deconvolution from the full width at half power (FWHP) of the maps; $D$ and $S_{v}$ indicate respectively the linear diameter and the integrated flux over the whole emitting region.

In Fig. 2 we show the spectra of the $C^{34} S(2-1)$ and (5-4) lines obtained integrating the emission over the core, while the spectra of the ground state and vibrationally excited $\mathrm{CH}_{3} \mathrm{OH}$ transitions are presented in Figs. 3 and 4. The most interesting feature is the fact that all lines observed with sufficiently high spectral resolution present asymmetric profiles, with a sharper cut-off on the blue-shifted side. One possibility is that this is due to red-shifted self-absorption, as suggested by the dip at $\sim-1 \mathrm{~km} \mathrm{~s}^{-1}$ in the $\mathrm{C}^{34} \mathrm{~S}(2-1)$ profile. We shall come back to this point in Sect. 4.3.
Table 3. Parameters of the continuum emission: $\Theta$ and $D$ are the deconvolved angular and linear diameters and $S_{v}$ is the integrated flux over the whole emitting region. A distance of $1.7 \mathrm{kpc}$ has been assumed.

\begin{tabular}{rcccc}
\hline \hline $\begin{array}{c}v \\
(\mathrm{GHz})\end{array}$ & $\begin{array}{c}\text { FWHP } \\
(\operatorname{arcsec})\end{array}$ & $\begin{array}{c}\Theta \\
(\operatorname{arcsec})\end{array}$ & $\begin{array}{c}D \\
(\mathrm{AU})\end{array}$ & $\begin{array}{c}S_{v} \\
(\mathrm{Jy})\end{array}$ \\
\hline 95.01 & 2.54 & 0.92 & 1570 & 0.022 \\
239.55 & 1.03 & 0.55 & 935 & 0.181 \\
\hline
\end{tabular}

Since one of the goals of the present study is to use $\mathrm{C}^{34} \mathrm{~S}$ to investigate the disk previously detected in $\mathrm{CH}_{3} \mathrm{CN}$ and $\mathrm{NH}_{3}$, it is worth comparing the distribution of the red- and blue-shifted $\mathrm{C}^{34} \mathrm{~S}$ emission with the bulk of the molecular gas traced by the millimeter continuum. This is done in Fig. 5 for both $\mathrm{C}^{34} \mathrm{~S}$ lines: there is little doubt that the gas is distributed in a NE-SW elongated structure consistent with the orientation $\left(\mathrm{PA}=53^{\circ} \pm\right.$ $8^{\circ}$ ) of the plane of the disk detected in $\mathrm{CH}_{3} \mathrm{CN}$ (see C99). A similar result holds for all transitions detected in this study, thus confirming that such a disk has a rich chemical composition.

On the other hand, the ground state $\mathrm{CH}_{3} \mathrm{OH}$ lines do not arise only from the disk, but also from the material ejected along the disk axis. This is shown in Fig. 6, where a map of the emission averaged under all of the $\mathrm{CH}_{3} \mathrm{OH}(2-1)$ ground state lines is compared to the jet revealed in the $\mathrm{SiO}(2-1)$ (from C99) and $\mathrm{H}_{2} v=0-1 \mathrm{~S}(1)$ transitions. It is interesting to note that Kurtz et al. (2004) have detected several $\mathrm{CH}_{3} \mathrm{OH}$ maser spots at $44 \mathrm{GHz}$ lying along the border of the thermal $\mathrm{CH}_{3} \mathrm{OH}$ emission in the jet, as shown in the upper panel of Fig. 6: this is consistent with the idea that class I methanol masers form in shocks. The $\mathrm{CH}_{3} \mathrm{OH}$ emitting regions seem complementary to those traced by the $\mathrm{SiO}$ and $\mathrm{H}_{2}$ molecules, although this effect is much more evident in the SE lobe than in the NW one. Such a complementarity resembles the distribution of the $\mathrm{NH}_{3}(3,3)$ and $(4,4)$ lines observed by Zhang et al. (1999; see their Fig. 1): as suggested by these authors, this result might be an effect of dissociation of the $\mathrm{CH}_{3} \mathrm{OH}$ and $\mathrm{NH}_{3}$ molecules in a high-velocity shock, which instead favours the formation of $\mathrm{SiO}$. Such a scenario is also consistent with the lower velocities measured in the $\mathrm{CH}_{3} \mathrm{OH}$ lines (as discussed in Sect. 4.2) with respect to those estimated by $\mathrm{C} 99$ for the $\mathrm{SiO}$ transition. 


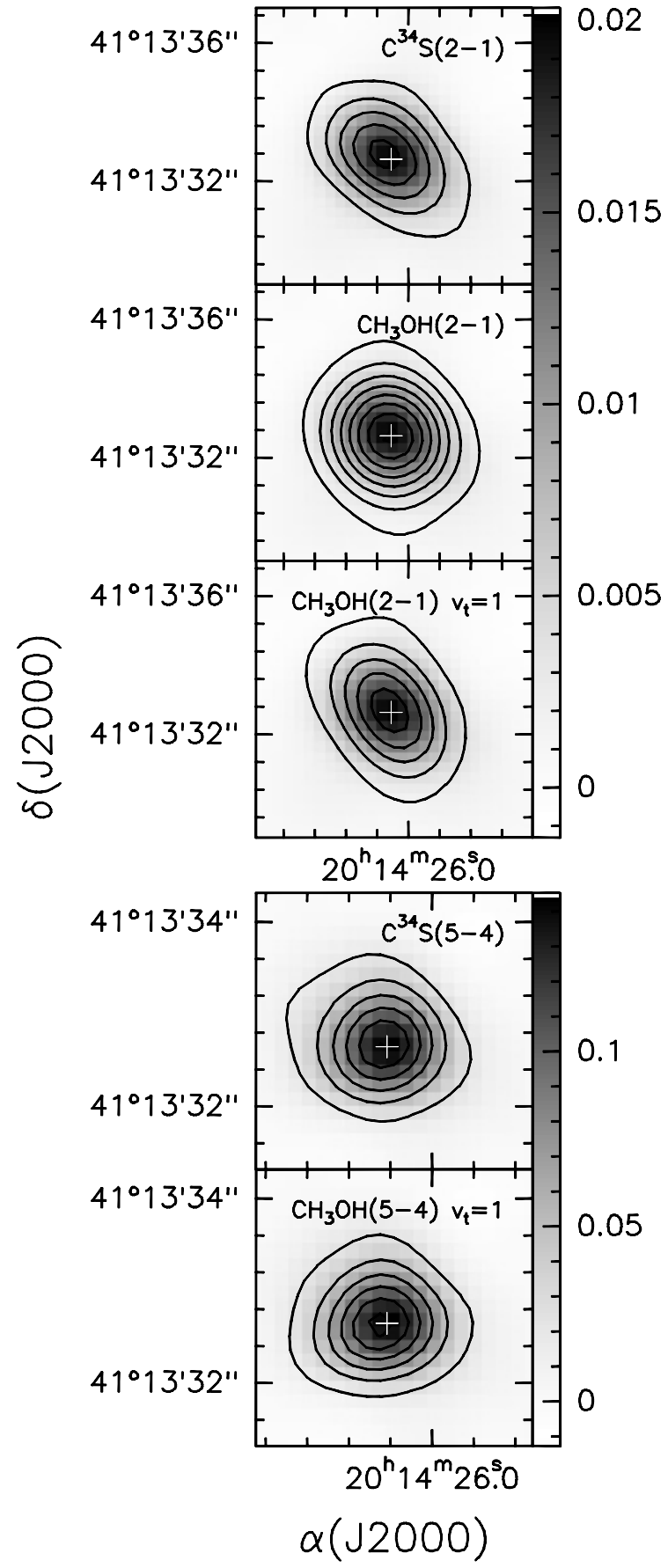

Fig. 1. Contour maps of the $\mathrm{C}^{34} \mathrm{~S}$ and $\mathrm{CH}_{3} \mathrm{OH}$ emission integrated under the line for the transitions detected towards IRAS 20126+4104 in the PdBI observations. Also shown are the maps of the millimeter continuum at the corresponding frequency (grey scale). The three $\mathrm{CH}_{3} \mathrm{OH}$ maps have been obtained averaging the emission under the ground state $\mathrm{CH}_{3} \mathrm{OH}(2-1)-1 \mathrm{E}, 0 \mathrm{~A}^{+}$, and $0 \mathrm{E}$ lines, the $\mathrm{CH}_{3} \mathrm{OH}(2-1)$ $v_{\mathrm{t}}=1+1 \mathrm{E}$ and $0 \mathrm{E}$ lines, and the $\mathrm{CH}_{3} \mathrm{OH}(5-4) v_{\mathrm{t}}=14 \mathrm{~A},-3 \mathrm{E},-4 \mathrm{E}$, $-2 \mathrm{E}, 2 \mathrm{~A}^{+}, 2 \mathrm{~A}^{-}, 3 \mathrm{~A}, 1 \mathrm{E}, 0 \mathrm{E}$, and $2 \mathrm{E}$ lines. The cross marks the position of the millimeter continuum peak. Contour levels range from 0.02 to 0.11 by $0.015 \mathrm{Jy} /$ beam for $\mathrm{C}^{34} \mathrm{~S}(2-1)$, from 0.02 to 0.3 by $0.14 \mathrm{Jy} /$ beam for $\mathrm{CH}_{3} \mathrm{OH}(2-1)$, from 0.01 to 0.11 by $0.02 \mathrm{Jy} /$ beam for $\mathrm{CH}_{3} \mathrm{OH}(2-1) v_{\mathrm{t}}=1$, from 0.05 to 0.37 by $0.08 \mathrm{Jy} /$ beam for $\mathrm{C}^{34} \mathrm{~S}(5-$ 4), and from 0.08 to 0.73 by $0.13 \mathrm{Jy} /$ beam for $\mathrm{CH}_{3} \mathrm{OH}(5-4) v_{\mathrm{t}}=1$ The synthesised beam full widths at half power (position angles) are the following (from top to bottom): $2^{\prime \prime} 7 \times 22^{\prime \prime} 1\left(44^{\circ}\right), 2^{\prime \prime} .7 \times 2^{\prime \prime} .6\left(79^{\circ}\right)$, $3^{\prime \prime} 1 \times 22^{\prime \prime} 0\left(35^{\circ}\right), 0{ }^{\prime} 87 \times 00^{\prime} 75\left(136^{\circ}\right), 0{ }^{\prime} 87 \times 00^{\prime} .75\left(137^{\circ}\right)$.

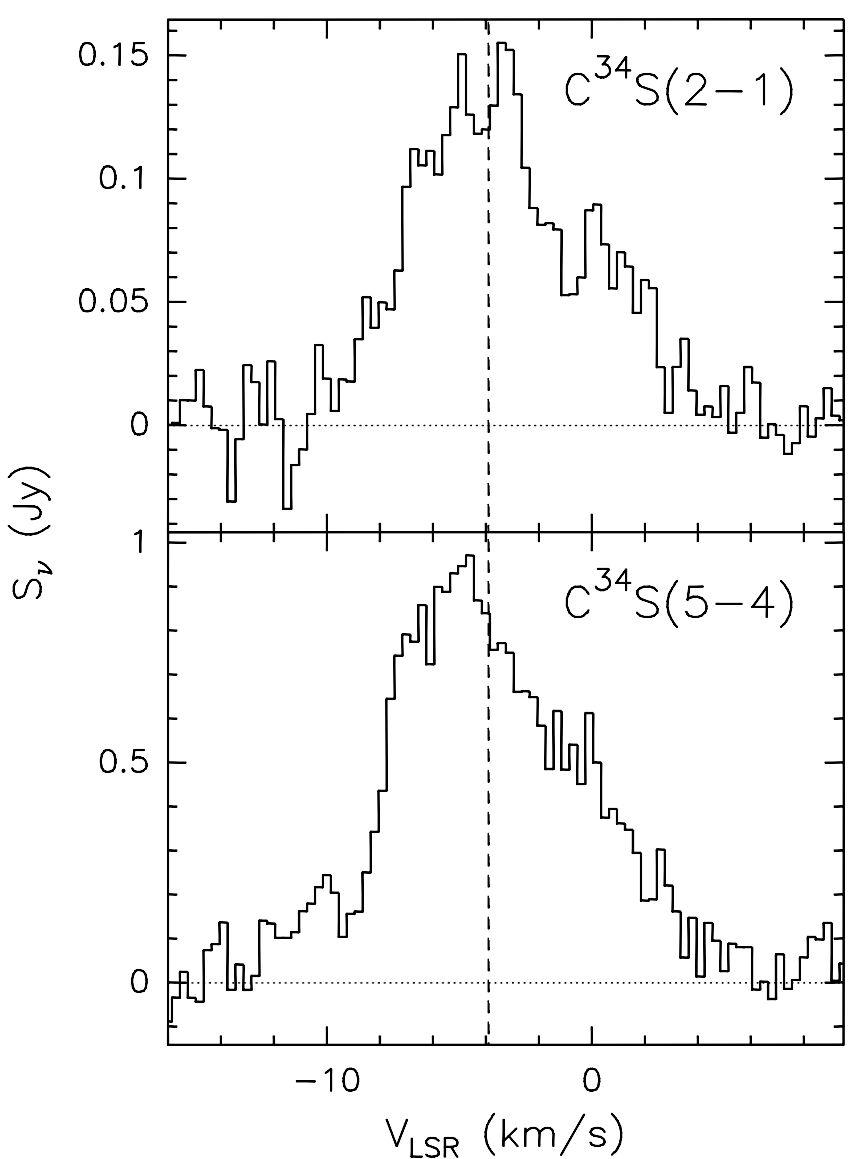

Fig. 2. Spectra obtained by integrating the $\mathrm{C}^{34} \mathrm{~S}$ line emission over the whole region where the corresponding transition is detected above a $3 \sigma$ level. The dashed and dotted lines indicate respectively the systemic velocity of the source and the zero flux level

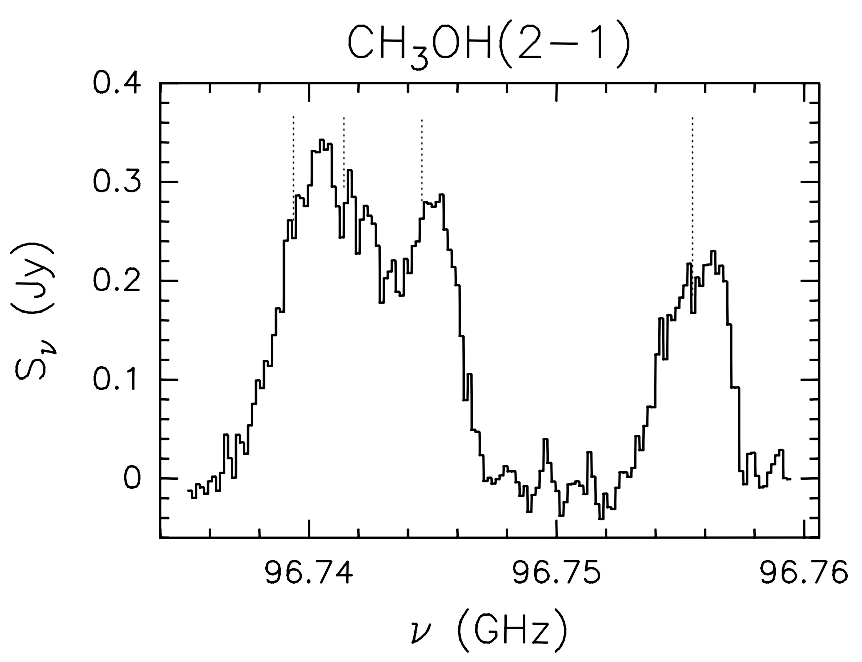

Fig. 3. Same as Fig. 2 for the ground state lines of $\mathrm{CH}_{3} \mathrm{OH}$. The dotted lines mark the frequencies of each $\mathrm{CH}_{3} \mathrm{OH}$ transition.

\section{Discussion}

In the following, we present an analysis of the disk-outflow system in IRAS 20126+4104 with the aim of giving an answer to the major questions left open by the previous studies of this object. On the basis of such studies one can identify three 


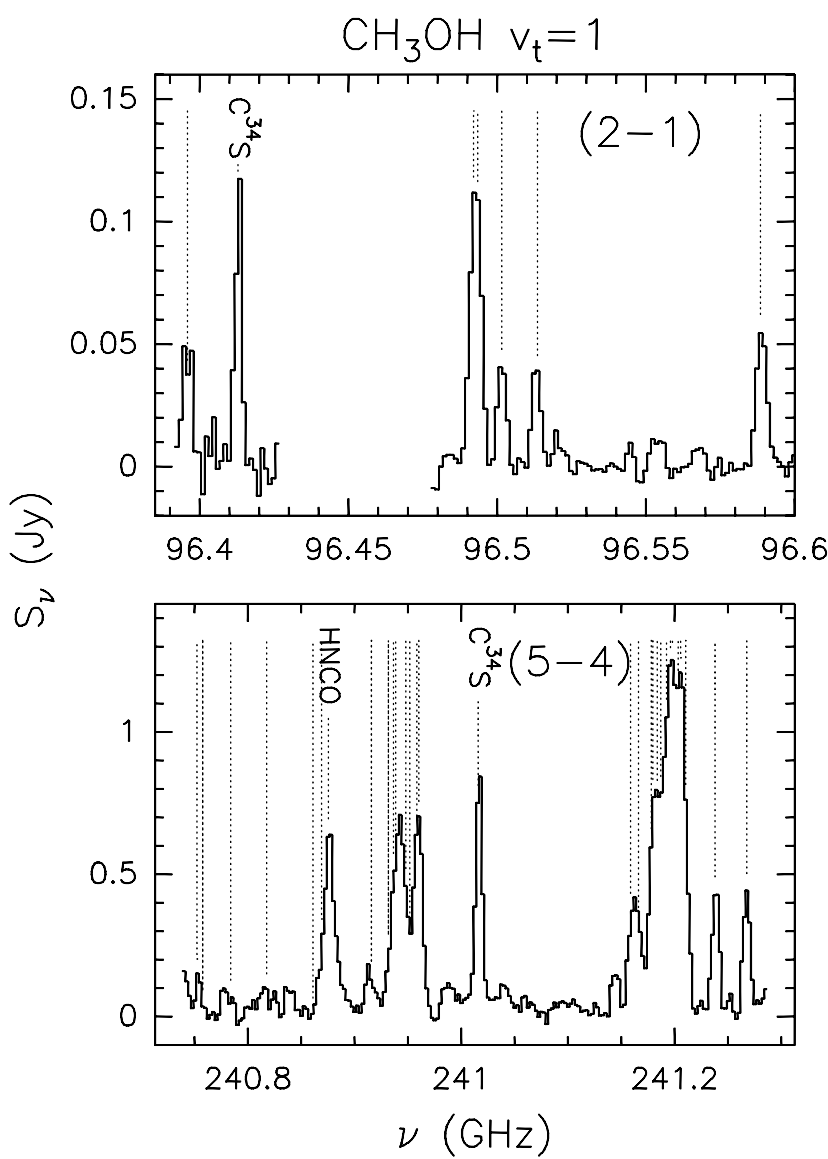

Fig. 4. Same as Fig. 3 for the vibrationally excited lines of $\mathrm{CH}_{3} \mathrm{OH}$.

components in this object: the disk $\left(\lessgtr 33^{\prime \prime} 5\right.$ or $\left.\lesssim 0.03 \mathrm{pc}\right)$, the outflow/jet, and the envelope ( $\$ 10$ or $0.08 \mathrm{pc}$ ). Since the main goal of our study is to investigate the former two (which will be discussed in Sects. 4.1 and 4.2), it is important to establish which is the contribution of each component to the tracers used in our analysis. For this purpose we adopt a criterion based on the kinematics and morphology of the emitting region. In fact, while all three components may contribute to the line emission close to the systemic velocity, in the line wings the contribution of the envelope is very likely negligible. Moreover, the direction of the velocity gradient in the disk is almost perpendicular to that in the outflow/jet, which makes it easy to discriminate between the two.

In practice, for a given line it will be sufficient to draw two position-velocity plots, one along the plane of the disk and the other along the axis of the flow, and check for the presence of velocity gradients in these two directions. For example, let us consider the case of $\mathrm{CH}_{3} \mathrm{OH}$. In Fig. 6 one can clearly see that the emission arises from both the outflow lobes and the central core. In Sects. 4.1 and 4.2, we shall demonstrate that a velocity gradient is found in both the outflow and the disk direction. We may hence conclude that $\mathrm{CH}_{3} \mathrm{OH}$ is tracing both the disk and the outflow component. The same method can be applied to all lines, leading to the conclusion that the disk is seen in $\mathrm{C}^{34} \mathrm{~S}, \mathrm{CH}_{3} \mathrm{CN}, \mathrm{CH}_{3} \mathrm{OH}$, and $\mathrm{HCO}^{+}$, whereas the outflow/jet is detected in $\mathrm{HCO}^{+}, \mathrm{CH}_{3} \mathrm{OH}$, and $\mathrm{SiO}$.

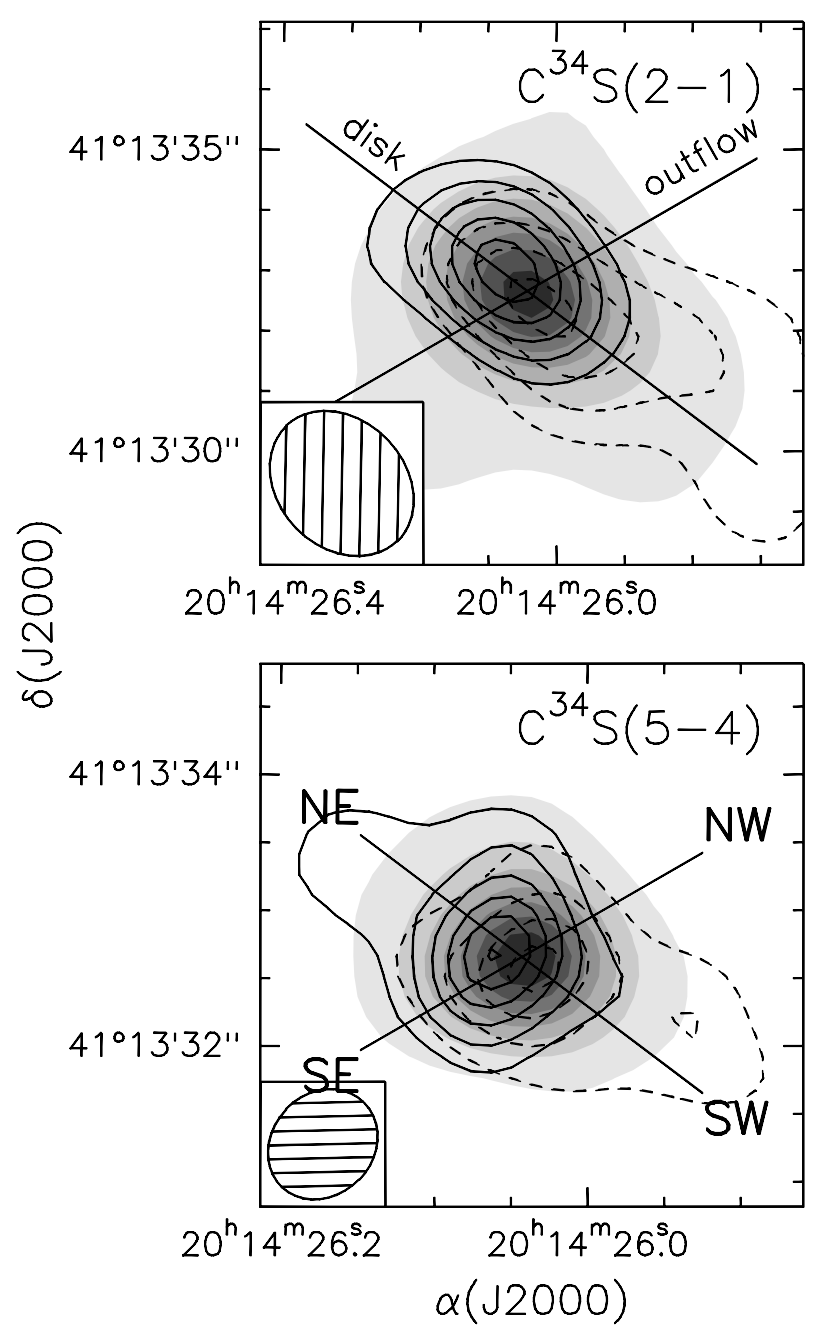

Fig. 5. Maps of the $\mathrm{C}^{34} \mathrm{~S}(2-1)$ (upper panel) and (5-4) (lower panel) emission integrated under the line wings superposed on the corresponding millimeter continuum maps (grey scale). Full and dashed contours refer respectively to blue- and red-shifted emission in a channel $0.3 \mathrm{~km} \mathrm{~s}^{-1}$ wide centred at -4.9 and $-3.1 \mathrm{~km} \mathrm{~s}^{-1}$ for $\mathrm{C}^{34} \mathrm{~S}(2-1)$, and -5.5 and $-2.5 \mathrm{~km} \mathrm{~s}^{-1}$ for $\mathrm{C}^{34} \mathrm{~S}(5-4)$. The SE-NW line marks the direction $\left(\mathrm{PA} \simeq-60^{\circ}\right.$ ) of the outflow axis (see C97 and C99), whereas the NE-SW line indicates the plane of the disk $\left(\mathrm{PA} \simeq 53^{\circ}\right)$ identified in C99.

What about the envelope? The distinction between this and the disk is not as sharp as that between the outflow and the disk because the disk may be reasonably seen as the innermost part of the envelope that has undergone flattening and rotation due to conservation of angular momentum. Nevertheless, for the purpose of simplifying the description of the environment of IRAS $20126+4104$, it is convenient to discriminate between the more quiescent material surrounding the disk and the rotating disk itself. In this simplified scenario, the envelope is important only at low velocities, where also part of the disk and outflow may significantly contribute. Morphologically, it must be located around the YSO position but this is also the case for the disk and - to some extent - for the outflow. It is hence very difficult to establish which fraction of the line or continuum emission is coming from the envelope, unless no 


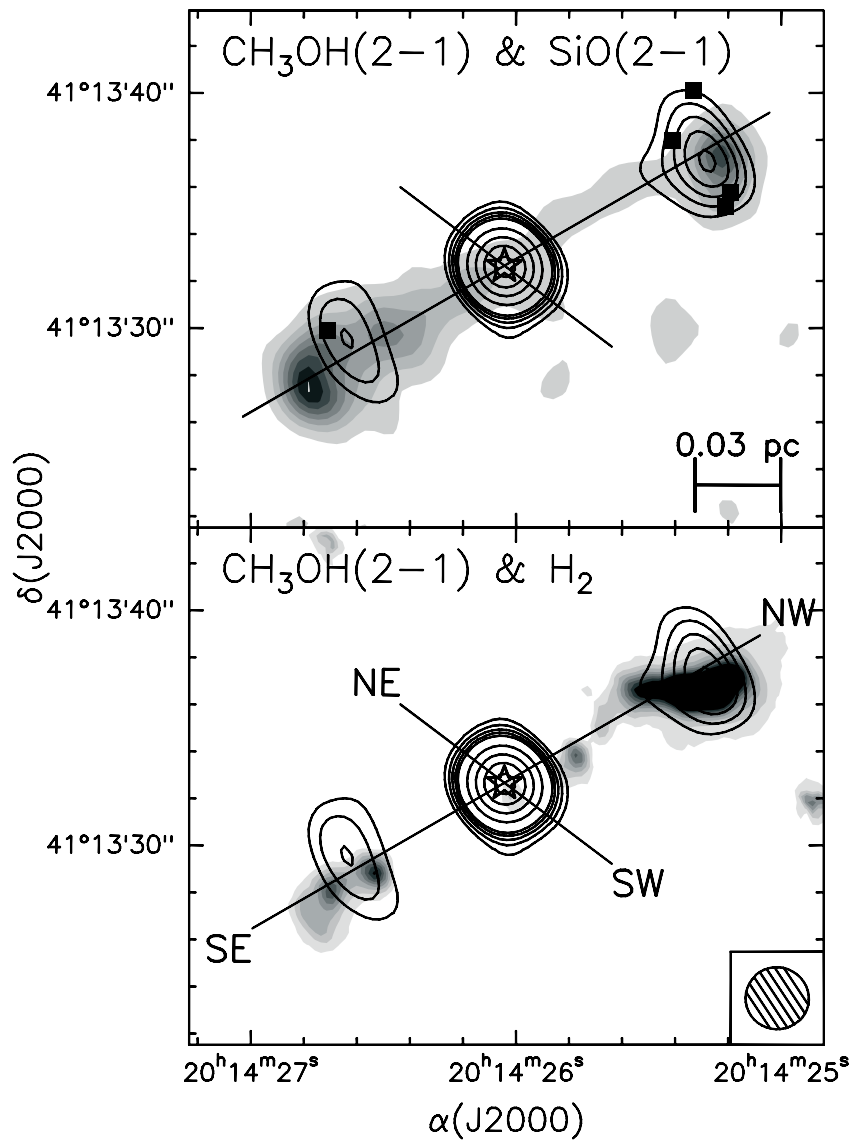

Fig. 6. Contour map of the $\mathrm{CH}_{3} \mathrm{OH}(2-1)$ emission averaged under all of the ground state lines, overlaied upon a grey-scale representation of the $\mathrm{SiO}(2-1)$ (upper panel; from C99) and $\mathrm{H}_{2} v=0-1 \mathrm{~S}(1)$ (lower panel) images. Contour levels range from 0.015 to 0.055 by $0.01 \mathrm{Jy} /$ beam and from 0.1 to 0.25 by $0.05 \mathrm{Jy} /$ beam. The SE-NW and NE-SW lines have the same meaning as in Fig. 5. The star marks the position of the $1 \mathrm{~mm}$ continuum peak, while the filled squares indicate the positions of the $7 \mathrm{~mm} \mathrm{CH}_{3} \mathrm{OH}$ maser spots mapped with the VLA by Kurtz et al. (2004); the error on the positions of the maser spots is 0 .' 6 . The synthesised beam of the $\mathrm{CH}_{3} \mathrm{OH}$ map is drawn in the bottom right of the lower panel.

emission is detected towards the YSO, as in the case of $\mathrm{SiO}$ (see top panel of Fig. 6) which may be taken as a "pure" outflow/jet tracer. Such a problem is even more important for the continuum emission for which the velocity information is obviously missing. This "confusion" between components in the central region is especially relevant for the disk, as the outflow extends over a much larger region. For a given tracer, it is hence very difficult to find out what fraction of the emission is arising from the disk and which from the envelope. We conclude that it is safer to consider any mass estimate of the disk as an upper limit.

\subsection{The disk}

As previously mentioned, evidence for a Keplerian disk in IRAS $20126+4104$ has been presented by various authors. In particular, the $\mathrm{CH}_{3} \mathrm{CN}$ data of $\mathrm{C} 99$ made it possible to obtain the direction of the plane of the disk by fitting the peak distribution in their Fig. 5b: the resulting position angle is $53^{\circ} \pm 7^{\circ}$, implying a position angle of $-37^{\circ} \pm 7^{\circ}$ for the disk axis. This is significantly different from the direction of the outflow/jet axis $\left(\mathrm{PA}=-60^{\circ}\right)$, obtained by the same authors from the $\mathrm{SiO}$ and $\mathrm{H}_{2}$ maps of the jet (see also Fig. 6).

The observations obtained so far were hindered by poor spectral or angular resolution, which prevented direct imaging of the disk geometry and velocity field, and hence an accurate estimate of the stellar mass. On the contrary, the new data obtained by us in the $\mathrm{C}^{34} \mathrm{~S}$ lines make it possible to resolve the disk both spatially and in velocity, as illustrated by the position-velocity plots in Fig. 7, where the offset is computed along the plane of the disk corresponding to the NE-SW line in Fig. 5. In the following (see Sect. 4.1.3) we present a model fit to these plots, but even a qualitative inspection of Fig. 7 is sufficient to show that our findings are in qualitative agreement with Keplerian rotation. For this purpose, we have drawn the borders of the region inside which emission is expected for an edge-on Keplerian disk of radius 4.'5 (0.037 pc) rotating about a $7 M_{\odot}$ star: this pattern agrees reasonably well with the data, taking into account that it is obtained for zero line width and infinite angular resolution.

In the light of these results, we have then re-examined the data in $\mathrm{C} 97$ and C99, which are compared to the new $\mathrm{C}^{34} \mathrm{~S}$ data in Figs. 8 and 9. In all cases the Keplerian pattern is evident, with the exception of the $\mathrm{CH}_{3} \mathrm{CN} \mathrm{K}=8$ and $\mathrm{CH}_{3} \mathrm{OH}$ lines, detected only over a region close to the position of the star. $\mathrm{HCO}^{+}$ and its isotopomer trace larger radii - and hence lower velocities - in the disk, with respect to the other molecules. This may be due to variations in the molecular abundances of the different species. Another possibility is that one is observing an excitation effect due to density and/or temperature gradients in the disk. A hint in this sense is given by the high energy (513 K) $\mathrm{CH}_{3} \mathrm{CN}(12-11) \mathrm{K}=8$ line (see Fig. 9) which, unlike the low energy (87-122 K) $K=2$ and 3 lines, presents two distinct peaks of emission: one close at the systemic velocity and close to the centre, the other slightly offset from the center and at $\sim 5 \mathrm{~km} \mathrm{~s}^{-1}$ with respect to the systemic velocity. The fact that a higher excitation transition arises from a small region close to the star supports the existence of a temperature increase towards the centre of the disk, as already suggested by C99. In the following section we investigate this possibility in some better detail and derive an estimate of the density and its distribution in the disk. On the other hand, the secondary peak in the $\mathrm{CH}_{3} \mathrm{CN}(12-11) K=8$ line could be due to a stellar companion of the (proto)star located at the centre of the disk. Although no other evidence of the existence of such a companion has been found, this cannot be ruled out a priori given that binary (or multiple) systems are more common than single stars. The presence of such a system could also explain the precession of the jet in IRAS 20126+4104 revealed by SYBT (see also Sect. 4.2.1). It is also worth noting that the secondary peak in the bottom panel of Fig. 9 lies on the Keplerian curve plotted in Fig. 7, which demonstrates that the velocity and position of such a peak are consistent with those of a lower mass companion orbiting around a $7 M_{\odot}$ star. 


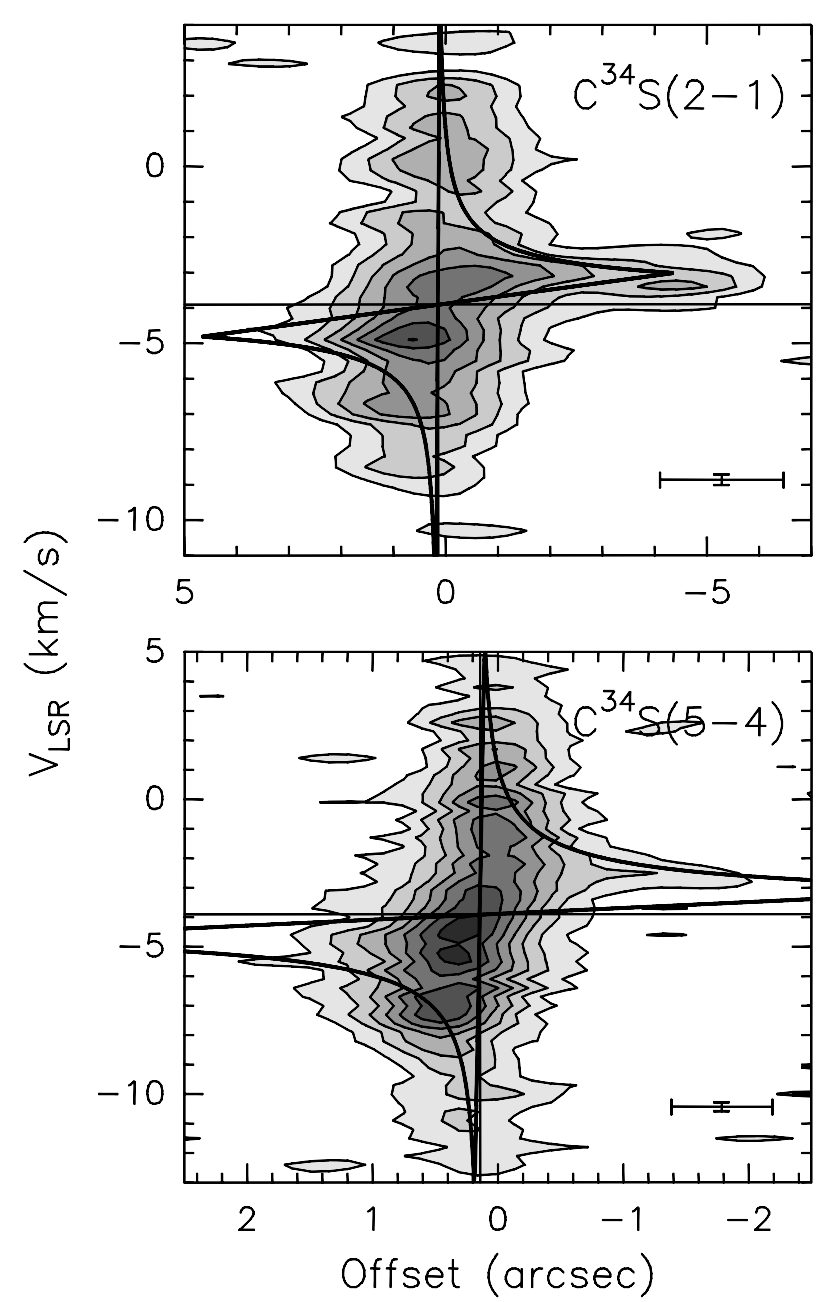

Fig. 7. Position-velocity plots along the plane of the disk (see Fig. 5) for the $\mathrm{C}^{34} \mathrm{~S}$ lines. The angular offset is positive towards NE and measured with respect to the phase centre of the observations. Contour levels range from 0.02 to 0.14 by $0.02 \mathrm{Jy} /$ beam for $\mathrm{C}^{34} \mathrm{~S}(2-1)$ and from 0.05 to 0.53 by $0.08 \mathrm{Jy} /$ beam for $\mathrm{C}^{34} \mathrm{~S}(5-4)$. The cross in the lower right of each panel gives the angular and spectral resolution of the corresponding map. The thick solid line marks the border of the region where emission is expected for an edge-on Keplerian disk of radius $4 . .5(0.037 \mathrm{pc})$ rotating about a $7 M_{\odot}$ point source. The vertical and horizontal straight lines mark respectively the position of the $1.3 \mathrm{~mm}$ continuum peak and the systemic velocity.

\subsubsection{Temperature and density structure}

A reasonable approximation to the kinetic temperature is represented by the rotation temperature obtained from the ratio between different lines of a thermalised molecular species. In the disk plane the density is large enough (see below) that $\mathrm{CH}_{3} \mathrm{OH}$ may be assumed in LTE, hence an estimate of the mean temperature of the disk can be derived from a rotation diagram of all the $\mathrm{CH}_{3} \mathrm{OH}$ lines detected (see Fig. 10): this gives a temperature of $170 \mathrm{~K}$ and a source averaged column density of $3 \times 10^{16} \mathrm{~cm}^{-2}$ for the $\mathrm{CH}_{3} \mathrm{OH}$ molecules.

A more detailed picture of the temperature is obtained from the ratio between the ground state $2{ }_{1}-1_{1} \mathrm{E}$ transition at $96755.508 \mathrm{MHz}$ and the vibrationally excited $2_{1}-1_{1} \mathrm{~A}^{-}$line at $96588.602 \mathrm{MHz}$. This choice has been dictated by the fact that this pair of lines is the only one to satisfy three basic requirements: high spectral resolution, large difference in energy $(311 \mathrm{~K})$, and no overlap in frequency with other lines.

The line profiles integrated over the disk are shown in Fig. 11a, whereas in Fig. 11b the rotation temperature computed at each velocity from the ratio of such profiles is presented. The most remarkable feature is the presence of a dip in the $v_{\mathrm{t}}=1$ transition in correspondence to the peak of the ground state line. It seems unlikely that this is due to self-absorption, as too large a $\mathrm{CH}_{3} \mathrm{OH}$ column density $\left(>4 \times 10^{19} \mathrm{~cm}^{-2}\right)$ would be needed for the $v_{\mathrm{t}}=11 \mathrm{~A}^{-}$transition to be optically thick. A more plausible explanation is that the dip is due to the temperature increase towards the centre of the disk: at low velocities (i.e. around the systemic velocity of $\sim-3.9 \mathrm{~km} \mathrm{~s}^{-1}$ ) the line emission comes from the outer radii, where the gas is colder and the ground state lines are more excited than the high-energy transitions; vice versa, at high velocities the emission originates close to the star, where the gas is hotter and the $v_{\mathrm{t}}=1$ line is favoured with respect to the ground state one. This can be seen in the position-velocity diagram in Fig. 12a, which plots the rotation temperature computed from the same two lines. Clearly, the maximum value is attained at high velocities (i.e. small radii), while the temperature drops at low velocities (i.e. large radii).

A quantitative estimate of the temperature gradient can be derived by fitting the rotation temperature profile in Fig. 11b. Under the assumption that one is observing a Keplerian disk seen edge-on and that the kinetic temperature $T$ depends on the radius $R$ as $T=T_{0}\left(R / R_{0}\right)^{q}$, it is possible to demonstrate (see Appendix A) that the mean temperature over the disk measured at a given velocity $v$ is

$$
\langle T\rangle_{v}=\frac{3 T_{0}}{2 q+3}\left(\min \left\{1,\left(\frac{v_{0}}{|v|}\right)^{3}\right\}\right)^{\frac{2}{3} q}
$$

with $v_{0}$ rotation velocity at radius $R_{0}$. This expression may be fitted to the curve in Fig. 11b: the best fit, represented by the dashed line in the same figure, is obtained for $T_{0}=67 \mathrm{~K}$, $v_{0}=2.3 \mathrm{~km} \mathrm{~s}^{-1}$, and $q=-0.57$. For a geometrically thin accretion disk, no matter whether passive (i.e. heated by the central star) or active (i.e. heated internally by viscosity), theory has established that the temperature profile is a power law with $q=-0.75$ (Pringle 1981): the latter value is consistent with that found by us, within the uncertainties of our method.

We also address the problem of the density distribution in the disk. The ratio between the $\mathrm{C}^{34} \mathrm{~S}(5-4)$ and (2-1) lines can be used to calculate the $\mathrm{H}_{2}$ density, as explained, e.g., in Cesaroni et al. (1991). Figure 12b shows a position-velocity plot of such a ratio along the plane of the disk, obtained after smoothing the $\mathrm{C}^{34} \mathrm{~S}(5-4)$ data to the same angular resolution as the (2-1) line. In order to convert this into a density estimate, we used the LVG code of Cesaroni et al. (1991), adopting a $\mathrm{C}^{34} \mathrm{~S}$ abundance relative to $\mathrm{H}_{2}$ of $5 \times 10^{-10}$, a velocity gradient of $13 \mathrm{~km} \mathrm{~s}^{-1} \mathrm{pc}^{-1}$ (equal to the ratio between the line width and the disk diameter), and a kinetic temperature of $170 \mathrm{~K}$ (obtained from $\mathrm{CH}_{3} \mathrm{OH}$ ). In general for any given temperature and line ratio, two distinct values of the density are possible. This is illustrated in Fig. 13, where such a ratio is plotted as a function 


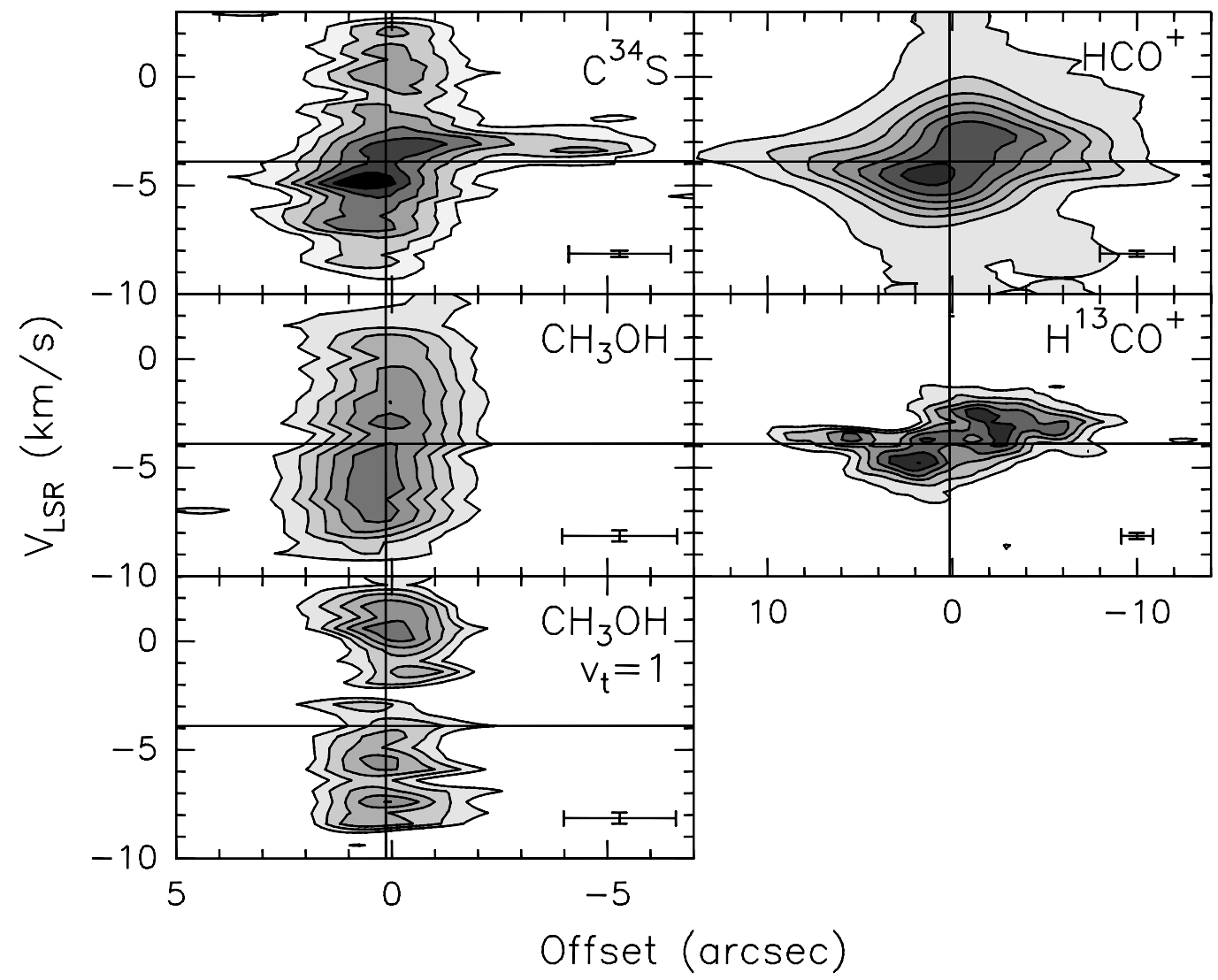

Fig. 8. Same as Fig. 7 for the $\mathrm{C}^{34} \mathrm{~S}(2-1), \mathrm{CH}_{3} \mathrm{OH}\left(2_{1}-1_{1}\right) \mathrm{E}, \mathrm{CH}_{3} \mathrm{OH}\left(2_{1}-1_{1}\right) v_{\mathrm{t}}=1 \mathrm{~A}^{-}, \mathrm{HCO}^{+}(1-0)$ (from C97), and $\mathrm{H}^{13} \mathrm{CO}^{+}(1-0)($ from $\mathrm{C} 99)$ lines. Contour levels range from 0.02 to 0.14 by $0.02 \mathrm{Jy} /$ beam for $\mathrm{C}^{34} \mathrm{~S}$, from 0.035 to 0.28 by $0.035 \mathrm{Jy} /$ beam for $\mathrm{CH}_{3} \mathrm{OH}$, from 0.02 to 0.06 by $0.01 \mathrm{Jy} /$ beam for $\mathrm{CH}_{3} \mathrm{OH} v_{\mathrm{t}}=1$, from 0.08 to 3.08 by $0.5 \mathrm{Jy} /$ beam for $\mathrm{HCO}^{+}$, and from 0.012 to 0.087 by $0.015 \mathrm{Jy} /$ beam for $\mathrm{H}^{13} \mathrm{CO}^{+}$.

of temperature and density. For instance, one can see that, for $T_{K}=170 \mathrm{~K}$, a line ratio of $1-$ the mean value in Fig. $12 \mathrm{~b}-$ is attained for either $n_{\mathrm{H}_{2}} \simeq 2.5 \times 10^{5} \mathrm{~cm}^{-3}$ or $\gtrsim 2 \times 10^{8} \mathrm{~cm}^{-3}$. Inspection of Fig. 12b suggests which of the two values must be chosen. As already pointed out for the temperature plot in Fig. 12a, going from low to high velocities corresponds to moving from the outer to the inner regions in the disk: correspondingly, the line ratio increases from $\sim 1$, to a maximum of $\sim 1.8$, and eventually decreases to $\sim 1$ at high velocities. Very likely this means that the density increases steadily outside in, starting from $\sim 2.5 \times 10^{5} \mathrm{~cm}^{-3}$ at the outer radius and reaching values $\gtrsim 2 \times 10^{8} \mathrm{~cm}^{-3}$ close to the centre.

\subsubsection{Mass}

The mass of the disk can be derived from its millimeter continuum emission. Such an estimate depends on the temperature of the dust and the composition of the grains. In order to evaluate the best possible value of the mass, we have considered all interferometric measurements available in the literature at millimeter wavelengths, where the emission is optically thin: these are plotted in Fig. 14 as a function of frequency. Note that the scatter of the values at $1 \mathrm{~mm}$ is resolved if only the uv-points in common between the different data sets are used for the image reconstruction. The spectrum in this regime can be described by a power law with index $\sim 2.7$, which implies a dust absorption coefficient $\kappa \propto v^{0.7}$. Under the assumption of thermal equilibrium between gas and dust at $170 \mathrm{~K}$ (see Fig. 10), one obtains a mass ranging from 0.65 to $3.6 M_{\odot}$ depending whether one adopts the dust opacities of Hildebrand (1983), Ossenkopf \& Henning (1994), or Kramer et al. (2003).

An independent estimate of the mass is obtained from the $\mathrm{CH}_{3} \mathrm{OH}$ column density integrated over the source itself, using the results of Sect. 4.1.1. In hot-core regions the $\mathrm{CH}_{3} \mathrm{OH}$ abundance relative to $\mathrm{H}_{2}$ is found to vary between $10^{-7}$ and $10^{-6}$ (see Van Dishoeck 1993). Consequently, one estimates a disk mass between 0.8 and $8 M_{\odot}$.

Given the large uncertainty on the $\mathrm{CH}_{3} \mathrm{OH}$ abundance, we consider more reliable the value obtained from the continuum emission, thus concluding that the mass of the disk within $\sim 5000$ AU from the star very likely lies in the range $1-4 M_{\odot}$.

\subsubsection{Velocity field and stellar mass}

As we mentioned earlier, the position-velocity plots of Fig. 7 represent solid evidence of Keplerian disk rotation and we now further discuss this critical point. In fact, the spectral and angular resolution in our observations were good enough to allow an accurate study of the velocity field inside the disk. This analysis is of fundamental importance to assess the correct value of the stellar mass and hence shed light on its nature and evolutionary phase. The basic procedure to determine the mass of the 


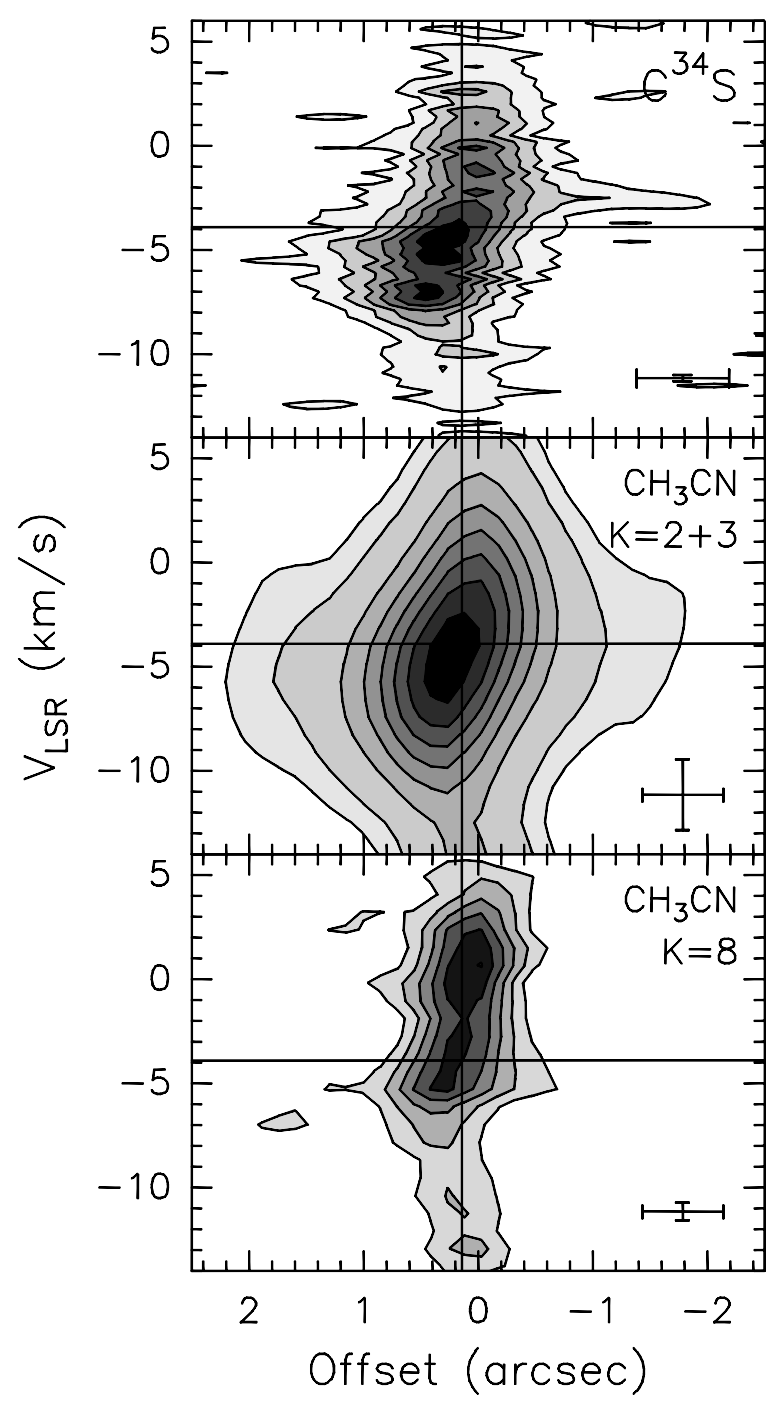

Fig. 9. Same as Fig. 7 for the $\mathrm{C}^{34} \mathrm{~S}(5-4)$ line, the $\mathrm{CH}_{3} \mathrm{CN}(12-11)$ average emission of the $K=2$ and 3 transitions, and the $\mathrm{CH}_{3} \mathrm{CN}(12-11)$ $K=8$ line. Note the different spectral resolution of the $\mathrm{CH}_{3} \mathrm{CN} K=$ $2+3$ lines. Contour levels range from 0.05 to 0.59 by $0.09 \mathrm{Jy} /$ beam for $\mathrm{C}^{34} \mathrm{~S}$, from 0.03 to 0.66 by $0.03 \mathrm{Jy} /$ beam for $\mathrm{CH}_{3} \mathrm{CN} K=2+3$, and from 0.035 to 0.16 by $0.025 \mathrm{Jy} /$ beam for $\mathrm{CH}_{3} \mathrm{CN} K=8$.

central star(s) consisted of using all of the data in the positionvelocity plot of Fig. 7 at once, by least-squares fitting the maps for several kinematical and physical parameters of the modeled keplerian disk.

In our model we consider an edge-on cylindrical disk undergoing Keplerian rotation. The disk has an outer, $R_{0}$, and inner, $R_{\mathrm{i}}$, radius, and the star is located at the center of the inner (cylindrical) cavity. The temperature and volume density are assumed to depend on the distance from the centre, $R$, respectively as $T \propto R^{q}$ and $n \propto R^{p}$. The radiative transfer equation was solved along a suitable number of lines of sight in the disk plane, taking into account the appropriate velocity coherence paths. Finally, the resulting line brightness temperature was convolved with the synthesized beam and with the velocity resolution of our PdBI observations, allowing the generation of a model position-velocity plot to be compared with the data.

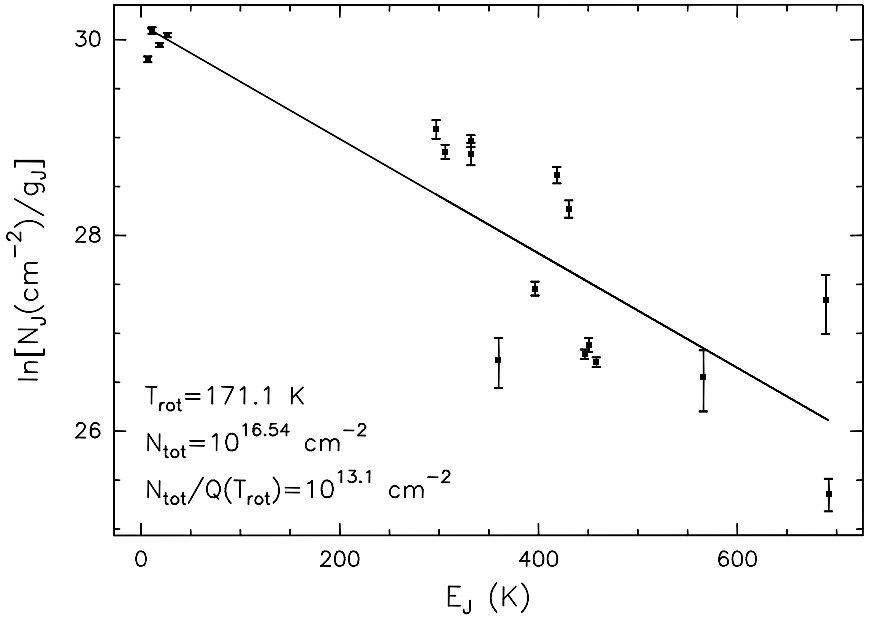

Fig. 10. Rotation diagram obtained from the average $\mathrm{CH}_{3} \mathrm{OH}(2-1)$ and (5-4) spectra over the disk. Ground state and vibrationally excited lines have been used. The straight line is a least square fit to the data corresponding to the rotational temperature and column density indicated in the bottom left. All lines above $200 \mathrm{~K}$ are $v_{\mathrm{t}}=1$ transitions, whose frequencies are listed below. $\mathrm{CH}_{3} \mathrm{OH}(2-1)$ $v_{\mathrm{t}}=1$ : 96396.008, 96492.203, 96493.594, 96501.656, 96513.656, 96553.781, 96588.602; $\mathrm{CH}_{3} \mathrm{OH}(5-4) v_{\mathrm{t}}=1: 240916.156,240958.797$, 240960.562, 241159.172, 241166.531, 241238.156, 241267.875.

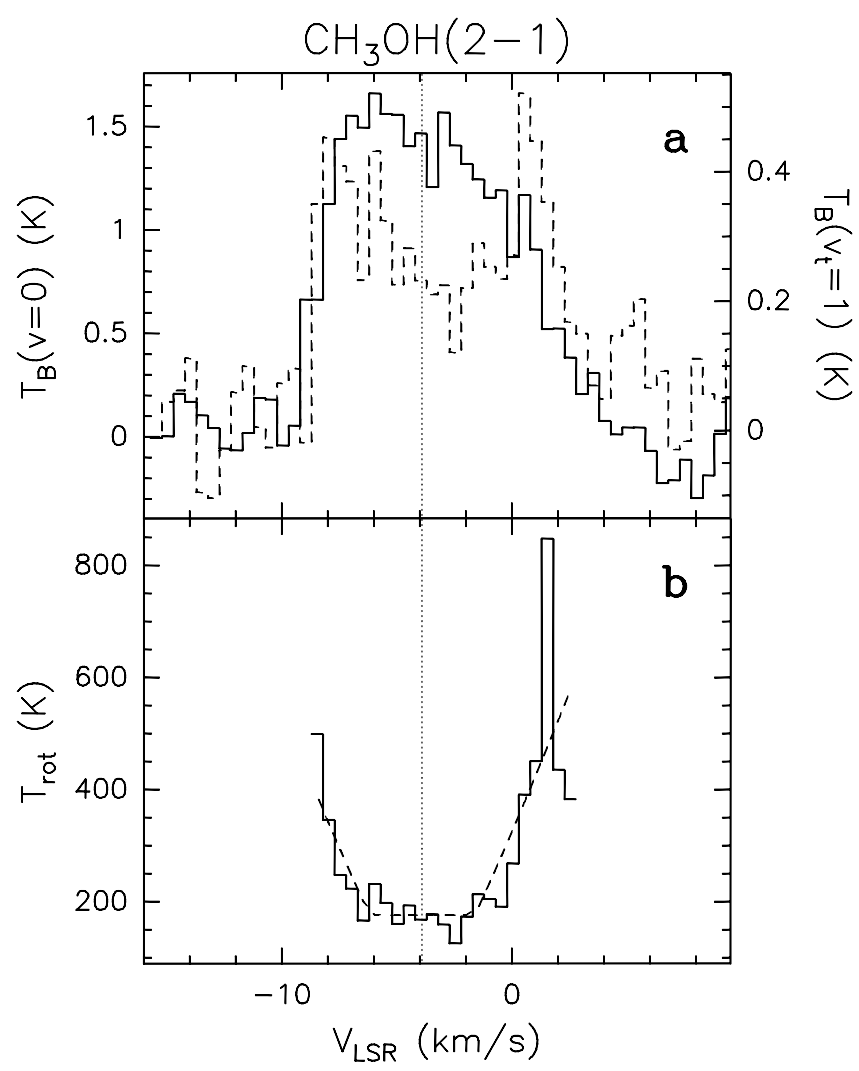

Fig. 11. a) Comparison between the spectra of the ground state $\mathrm{CH}_{3} \mathrm{OH}\left(2_{1}-1_{1}\right) \mathrm{E}$ transition (full histogram) and the vibrationally excited $\mathrm{CH}_{3} \mathrm{OH}\left(2_{1}-1_{1}\right) \mathrm{A}^{-}$line (dashed) obtained by averaging the emission over the disk. b) Rotation temperature as a function of LSR velocity, computed from the ratio of the two lines in the upper panel. The dashed line is the best fit obtained from Eq. (1). The dotted vertical line marks the systemic velocity. 


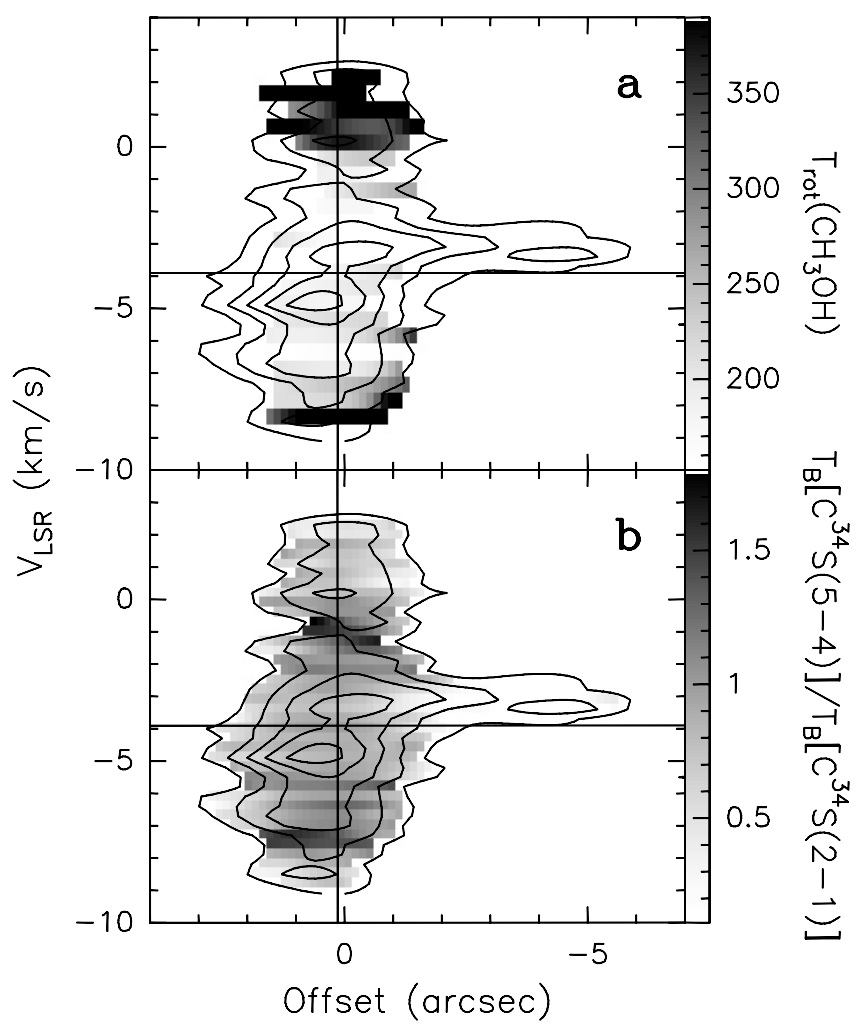

Fig. 12. a) Position-velocity plot along the plane of the disk for the rotation temperature (grey scale) obtained from the ratio between the same $\mathrm{CH}_{3} \mathrm{OH}(2-1)$ lines as in Fig. 11. For the sake of comparison, also a contour map of the $\mathrm{C}^{34} \mathrm{~S}(2-1)$ line emission is shown; contour levels range from 0.03 to 0.15 by $0.03 \mathrm{Jy} / \mathrm{beam}$. b) Same as above, for the ratio (grey scale) between the $C^{34} S(5-4)$ and $C^{34} S(2-1)$ lines.

The least-squares fitting of the map then followed three steps. First, the model data, obtained with the procedure outlined above, were resampled on the same grid points as the observed data. Then, for each selected mass of the central star(s) the least-squares procedure found the best-fit parameters. The rotation velocity at the outer radius is not an independent parameter but it is obtained from the star mass and $R_{0}$. The value of $R_{0}$ was assumed to be the same for both $\mathrm{C}^{34} \mathrm{~S}$ lines. Finally, several trial masses were used and we found that the best results were achieved for a mass of $7 M_{\odot}$. Besides the mass of the central star, the best-fit parameters obtained with this procedure are the line width $(\Delta V)$, the excitation temperature at the outer radius of the disk $\left(T_{\mathrm{ex}}\right)$, the peak column density of the $\mathrm{C}^{34} \mathrm{~S}$ molecule $\left(N_{\mathrm{C}^{34} \mathrm{~S}}\right)$, and the power-law index for the density gradient $(p)$. The power-law index for the temperature profile was instead fixed at $q=-0.75$ (see Sect. 4.1.1). The best fits have been obtained for the parameters listed in Table 4 and are compared to the data in Fig. 15.

Although the pattern is reproduced reasonably well, both in the overall shape and intensity, a few caveats are in order. First of all, $\mathrm{C}^{34} \mathrm{~S}$ is unlikely to be in LTE, so that the values of $T_{\text {ex }}$ and $N_{\text {mol }}$ used in the computation are to be regarded as a way to parameterise the excitation conditions and not as true physical parameters. This explains why the fit to the $C^{34} S(2-1)$ line is obtained for slightly different parameters than that to the $\mathrm{C}^{34} \mathrm{~S}(5-4)$ line. Moreover, the density gradient describes the

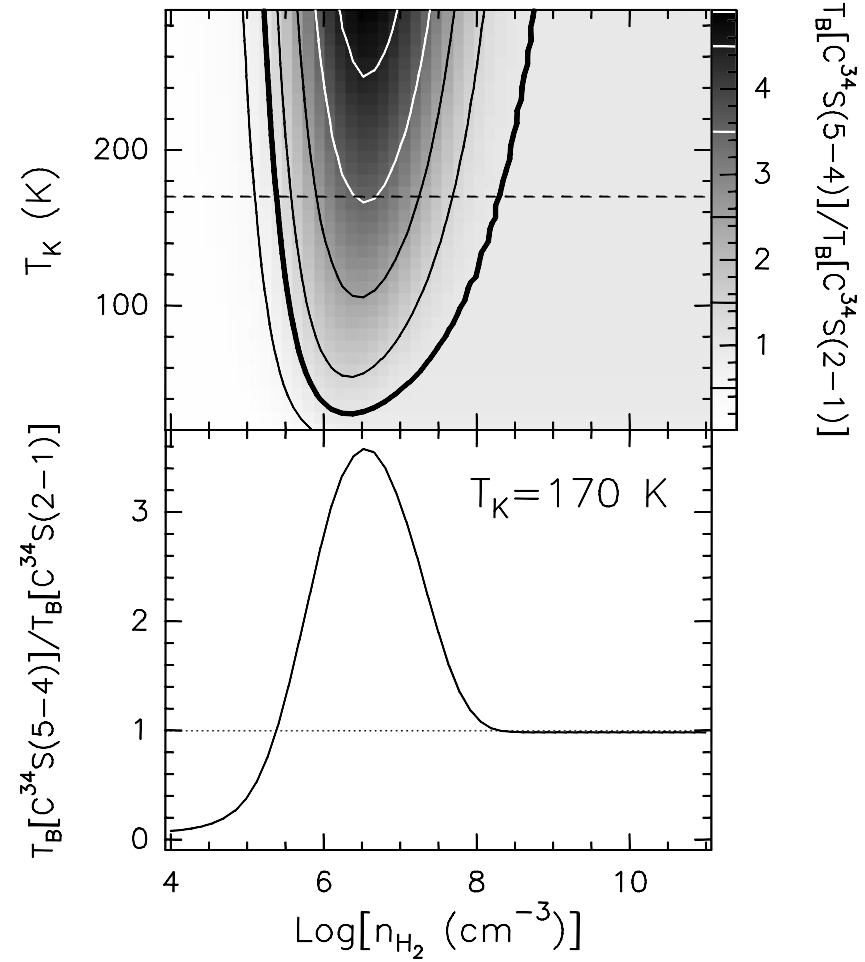

Fig. 13. Top panel: plot of the britghtness temperature ratio between the $\mathrm{C}^{34} \mathrm{~S}(5-4)$ and (2-1) lines computed with the LVG code by Cesaroni et al. (1991) assuming a $\mathrm{C}^{34} \mathrm{~S}$ abundance relative to $\mathrm{H}_{2}$ of $5 \times 10^{-10}$ and a velocity gradient of $13 \mathrm{~km} \mathrm{~s}^{-1} \mathrm{pc}^{-1}$. The values of the thin contour levels are indicated in the scale to the right, while the thick contour corresponds to a ratio of 1 . The dashed line indicates $T_{K}=170 \mathrm{~K}$, the rotational temperature obtained from $\mathrm{CH}_{3} \mathrm{OH}$ (see Fig. 10). Bottom panel: same ratio as above, as a function of density for a fixed temperature of $170 \mathrm{~K}$. The dotted line corresponds to a ratio of 1 .

Table 4. Parameters for the model fits in Fig. 15.

\begin{tabular}{lccc}
\hline \hline Parameter & $\mathrm{HCO}^{+}(1-0)$ & $\mathrm{C}^{34} \mathrm{~S}(2-1)$ & $\mathrm{C}^{34} \mathrm{~S}(5-4)$ \\
\hline$M_{*}\left(M_{\odot}\right)$ & 12 & 7 & 7 \\
$R_{0}(\operatorname{arcsec})$ & 7.1 & 3.5 & 3.5 \\
$\Delta V\left(\mathrm{~km} \mathrm{~s}^{-1}\right)$ & 0.40 & 0.40 & 0.45 \\
$T_{\text {ex }}(\mathrm{K})$ & 50 & 40 & 30 \\
$p$ & -2.1 & -3.0 & -2.8 \\
$N_{\text {mol }}\left(10^{12} \mathrm{~cm}^{-2}\right)$ & 0.22 & 17 & 8.4 \\
\hline
\end{tabular}

variation of the $\mathrm{C}^{34} \mathrm{~S}$ density, which does not necessarily resembles the $\mathrm{H}_{2}$ density change if the $\mathrm{C}^{34} \mathrm{~S}$ abundance depends on the radius. Due to these limitations, most of the physical quantities obtained from the fits are to be regarded with caution and are certainly affected by large errors. The sole exception is represented by the mass of the star, which is quite well constrained by the shape of the position-velocity plots: in particular, a small variation in the velocity of the "spike" corresponding to the largest radius where emission is detected will cause a significant change in the stellar mass. One may thus estimate the uncertainty on the value of the mass, assuming an error of $0.15 \mathrm{~km} \mathrm{~s}^{-1}$ (half the spectral channel used in the positionvelocity plots) and 0.'4 (half the beam size at $1.3 \mathrm{~mm}$ ): for a 


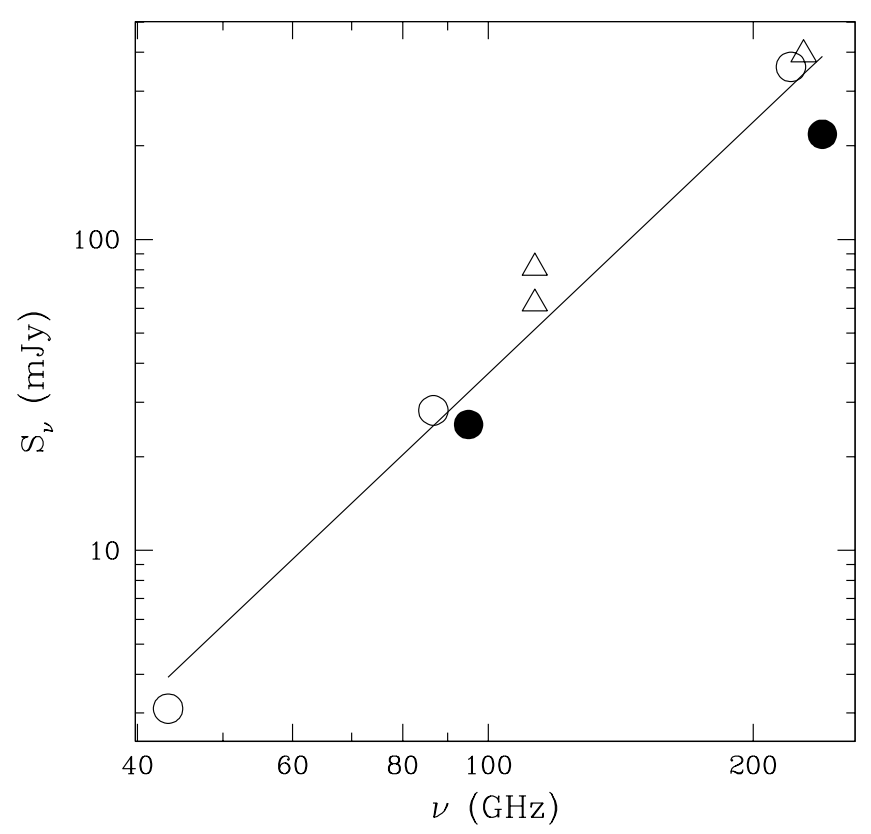

Fig. 14. Spectrum of the continuum emission measured in interferometric observations towards IRAS 20126+4104. Filled points refer to the present study; empty circles to the measurements of C97, C99, and Hofner et al. (1999); and empty triangles to the data of SYBT. The straight line is a least square fit to the data corresponding to $S_{v}(\mathrm{mJy})=1.55 \times 10^{-4}[v(\mathrm{GHz})]^{2.69}$.

Keplerian orbit passing through a point at $2.2 \pm 0.4$ with velocity $1.3 \pm 0.15 \mathrm{~km} \mathrm{~s}^{-1}$, the resulting central mass is $7 \pm 2 M_{\odot}$.

This result significantly differs from the estimate of $\sim 20 M_{\odot}$ obtained by C99 and Zhang et al. (1998). The method used by the C99 to calculate the mass of the star is based on rough estimates of the disk diameter and corresponding velocity dispersion, severely affected by the poor spectral resolution available ( $\sim 10$ times worse than in the present study): one may get an idea of this by comparing the top with the middle panel in Fig. 9. On the other hand, the observations by Zhang et al. (1998) did not suffer by this problem: both angular and spectral resolution are sufficient to resolve the Keplerian pattern (see their Fig. 3) and their model fit seems to reproduce the data reasonably well with a point mass of $20 M_{\odot}$. Interestingly, a similar result is obtained from our $\mathrm{HCO}^{+}$data (see Fig. 8). Applying our numerical model to such plots (see Fig. 15), we find a reasonable fit for a stellar mass of about $12 M_{\odot}$, significantly greater than that needed to fit the $\mathrm{C}^{34} \mathrm{~S}$ data.

However, both the ammonia maps of Zhang et al. (1998) and the $\mathrm{HCO}^{+}$maps of $\mathrm{C} 97$ and $\mathrm{C} 99$ sample regions at least twice as large as that sampled by our $\mathrm{C}^{34} \mathrm{~S}$ observations: this suggests that part of the mass required by the "Keplerian" fits to the outer disk might be contributed by the inner portion of the disk itself rather than by the star. In fact, Bertin \& Lodato (1999) have recently demonstrated that the rotation curve of self-gravitating circumstellar disks follows a Keplerian pattern close to the star but tends to a constant velocity at larger distances, where the disk mass enclosed inside the radius becomes greater than the stellar mass. This might be the case of IRAS 20126+4104. In Sect. 4.1.2 we have estimated a mass of

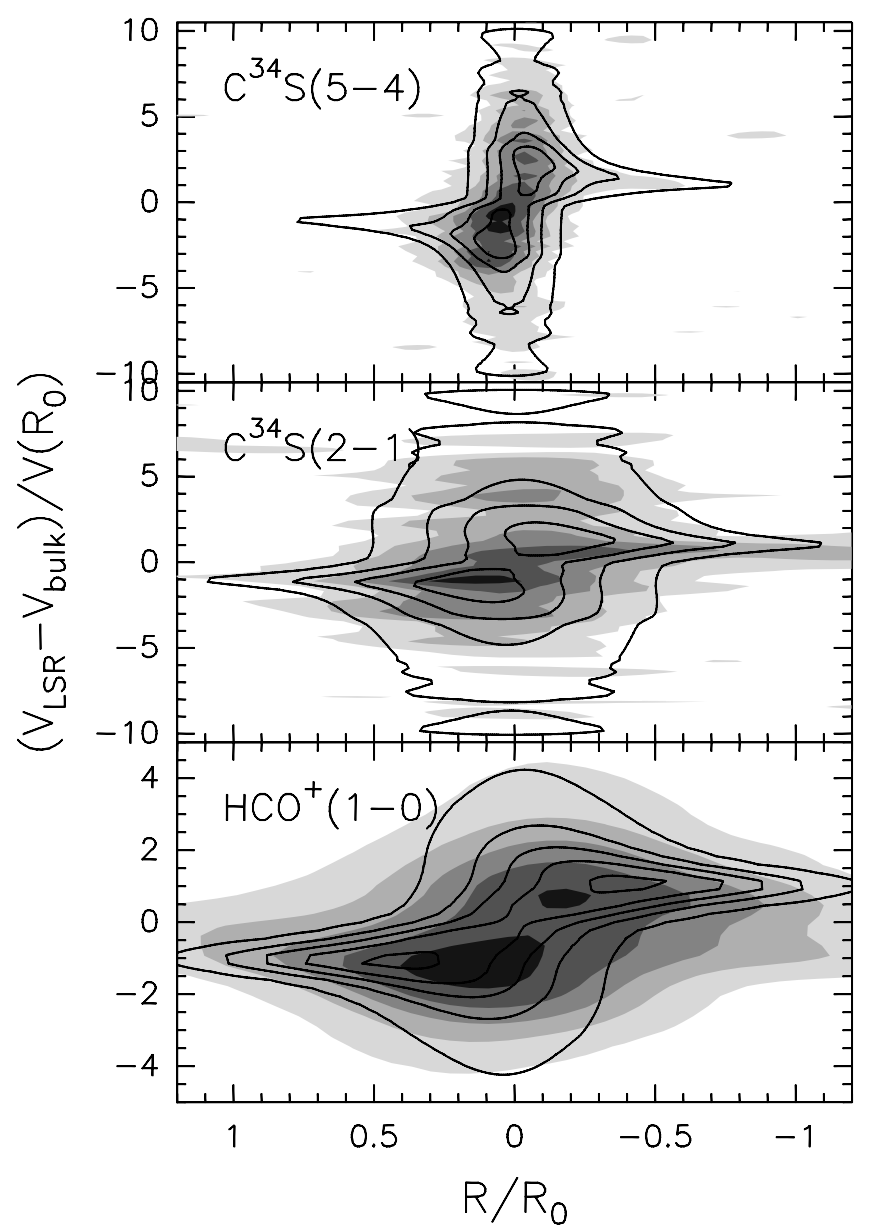

Fig. 15. Best-fit position-velocity plots along the disk plane obtained with the model described in the text, for $\mathrm{C}^{34} \mathrm{~S}(2-1)$ (top), $\mathrm{C}^{34} \mathrm{~S}(5-4)$ (middle) and $\mathrm{HCO}^{+}(1-0)$ (bottom). Contour levels are the same for the data and corresponding model and are drawn at 10,30, 50, 70, and $90 \%$ of the data peak value. The best-fit models for $\mathrm{C}^{34} \mathrm{~S}(2-1)$ and $\mathrm{C}^{34} \mathrm{~S}(5-4)$ have been obtained with a $7 M_{\odot}$ stellar mass, whereas the best-fit model of $\mathrm{HCO}^{+}(1-0)$ corresponds to a $12 M_{\odot}$ mass.

the disk of $\leq 4 M_{\odot}$ inside the $\mathrm{C}^{34} \mathrm{~S}$ region: this is significantly less than the stellar mass. However, inside a region twice as large, the disk mass may increase by a factor $\sim 4$, assuming constant column density: this brings the total mass of the star+disk system to $\leq 16 M_{\odot}$, i.e. closer to the estimate obtained from $\mathrm{NH}_{3}$ and $\mathrm{HCO}^{+}$.

In conclusion, we believe that our estimate of $\sim 7 M_{\odot}$ is a reasonable value for the mass of the central (proto)star.

\subsection{The outflow/jet}

The existence of a bipolar outflow/jet in IRAS 20126+4104 has been assessed by many authors in a variety of tracers over scales ranging from $100 \mathrm{AU}$ to $0.5 \mathrm{pc}$. Since the jet and the outflow are manifestations of the same ejection phenomenon, for the sake of simplicity in the following we shall use only the word "outflow" to indicate both the jet detected in $\mathrm{H}_{2}, \mathrm{SiO}$, $\mathrm{CH}_{3} \mathrm{OH}$, and $\mathrm{NH}_{3}$, and the outflow seen in $\mathrm{CO}, \mathrm{HCO}^{+}$, and their isotopomers. 
The knowledge about the outflow in IRAS 20126+4104 prior to the present study may be summarised as follows:

- The opening angle of the flow is $21^{\circ}$ and its axis forms an angle of $9^{\circ}$ with the plane of the sky, with the north-western part lying in the halfspace of the observer with respect to the plane of the sky (see C99).

- The position angle of the outflow axis remains equal to $\sim-60^{\circ}$ at a distance from the origin ranging from 0 '. $^{\prime}$ (170 AU) to 10" (0.08 pc) (Moscadelli et al. 2000; Hofner et al. 1999; Zhang et al. 1999; C97; C99; Kawamura et al. 1999 ), but becomes $\sim 0^{\circ}$ over a region of $\sim 1^{\prime}$, as demonstrated by SYBT, who interpret this as precession of the outflow.

- The expansion velocity of the flow lies in the range 20 $200 \mathrm{~km} \mathrm{~s}^{-1}$, depending on the distance from the origin, which suggests acceleration of the gas (see Moscadelli et al. 2000 and C99).

- A study of the emission at different velocities across the $\mathrm{HCO}^{+}$and $\mathrm{SiO}$ lines reveals that while the blue and red lobes are clearly separated at high velocities (i.e. in the outer wings of the line) with the blue-shifted emission located to the NW and the red-shifted to the SE, at low velocities the red- and blue-shifted lobes overlap (see C97 and C99).

The situation is outlined in Figs. 6 and 16. The latter presents the position-velocity plots along the outflow axis for the new $\mathrm{CH}_{3} \mathrm{OH}(2-1)$ data (contours) and the $\mathrm{HCO}^{+}(1-0)$ and $\mathrm{SiO}(2-$ 1) transitions (grey scales) observed by $\mathrm{C} 99$. The overlap between blue and red lobes mentioned above stands out clearly in this figure: while close to the systemic velocity no clear positional separation is seen between blue- and red-shifted emission, at high-velocities the red-shifted gas is present only to the SE and the blue-shifted to the NW. This effect has been convincingly explained by $\mathrm{C} 99$ with the fact that the outflow cone crosses the plane of the sky (see their Fig. A1 for a sketch of the model). The gas close to the plane of the sky moves almost perpendicularly to the line of sight and lies on both halfspaces with respect to the plane of the sky: consequently it is seen at low velocities and contributes to both red- and blue-shifted emission in the same lobe. The remaining material lies in only one halfspace and has a larger velocity component along the line of sight: it is hence seen only at either red- or blue-shifted high velocities.

However, this model cannot explain why the intensity of the low-velocity $\mathrm{HCO}^{+}$emission is not symmetric with respect to the systemic velocity (see upper panel in Fig. 16). In fact, the low-velocity pattern presents an S-shaped appearance so that the emission to the SE peaks at blue-shifted velocities, whereas that to the NW is clearly red-shifted. Such an asymmetry cannot be explained with the homogeneous conical model of the flow adopted by $\mathrm{C} 99$. Although less prominent, the same pattern is visible also in the position-velocity plots of $\mathrm{CH}_{3} \mathrm{OH}$ (see contours in Fig. 16) and $\mathrm{NH}_{3}$ (see Fig. 3 of Zhang et al. 1999).

An alternative to the position-velocity plot of Fig. 16 are the $\mathrm{HCO}^{+}$outflow maps presented in Fig. 17, where - following $\mathrm{C} 97$ - the emission in the inner wings of the line (low velocity) is compared to that in the outer wings (high velocity). Note

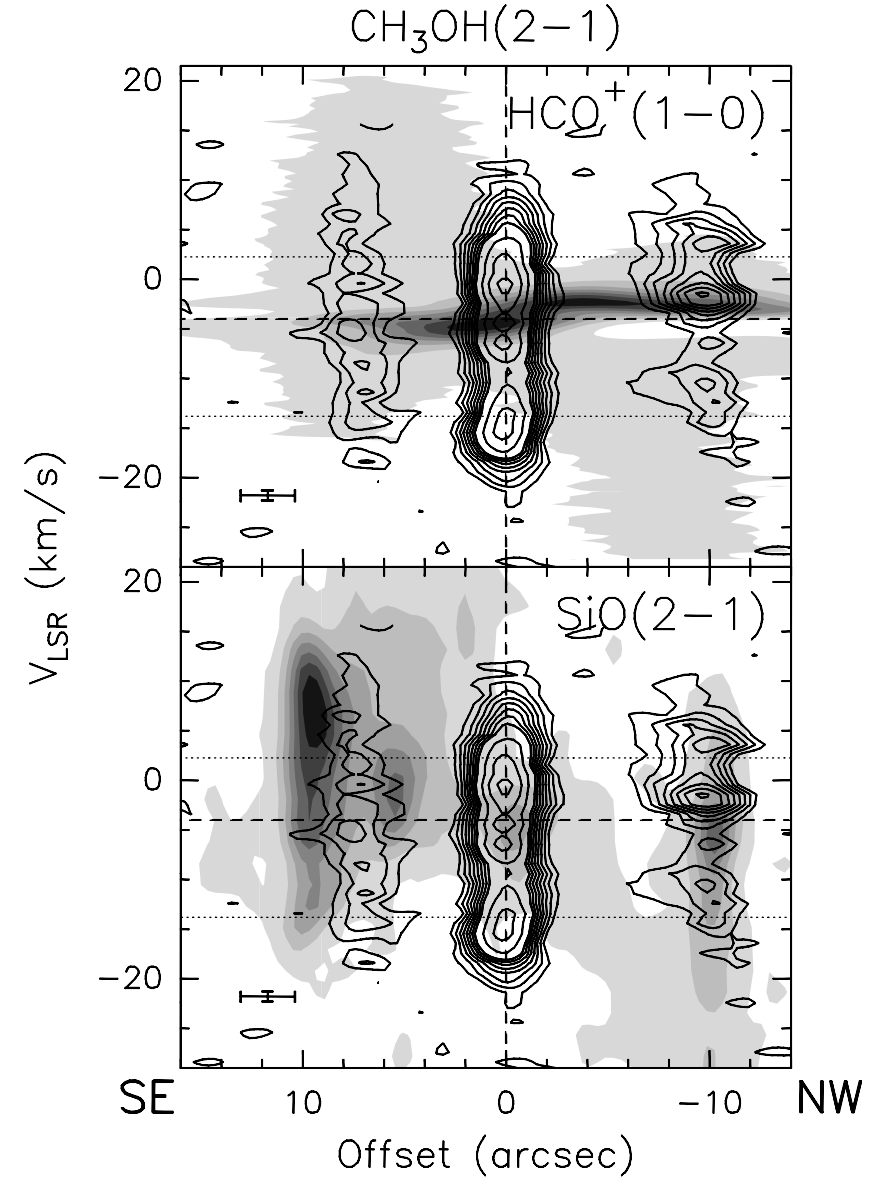

Fig. 16. Position-velocity plot along the outflow axis for the $\mathrm{CH}_{3} \mathrm{OH}(2-1)$ ground state lines (contours) overlaied to the $\mathrm{HCO}^{+}(1-0)$ and $\mathrm{SiO}(2-1)$ plots (grey scales) respectively from $\mathrm{C} 97$ and C99. The offset is measured positive towards SE and from the phase centre of the observations. The dashed vertical and horizontal lines indicate respectively the position of the $1 \mathrm{~mm}$ continuum peak and the systemic velocity. For $\mathrm{CH}_{3} \mathrm{OH}$ the latter refers to the $2_{0}-1_{0} \mathrm{~A}^{+}$ transition, whereas the dotted lines mark the positions of the $2_{-1}-1_{-1} \mathrm{E}$ and $2_{0}-1_{0} E$ lines. The contour levels range from 0.012 to 0.117 by $0.015 \mathrm{Jy} /$ beam and from 0.117 to 0.267 by $0.05 \mathrm{Jy} /$ beam.

that the maps have been rotated by $37^{\circ}$ to align the disk rotation axis to the $y$-axis of the figure. For the sake of comparison, also the $\mathrm{C}^{34} \mathrm{~S}$ disk and $\mathrm{SiO}$ flow are shown. The $\mathrm{S}$-shaped feature in the $\mathrm{HCO}^{+}$position-velocity plot has the consequence that the low-velocity gas is dominated by red-shfted emission in the NW lobe and by blue-shifted emission in the SE lobe, whereas in a homogeneous outflow the blue- and red-shifted gas should contribute almost equally to both lobes at low velocities.

A naïve explanation of this effect is that the S-shaped pattern is due to a second outflow with a different inclination with respect to the line of sight. Although it is impossible to rule out this possibility, we also present an alternative scenario which requires only one outflow and satisfies all the observational evidence collected so far. The key to interpret the S-shaped pattern in the $\mathrm{HCO}^{+}$position-velocity plot is the precession of the outflow discovered by SYBT. Therefore, before discussing the S-shaped feature, we elaborate a model to describe such a precession. 


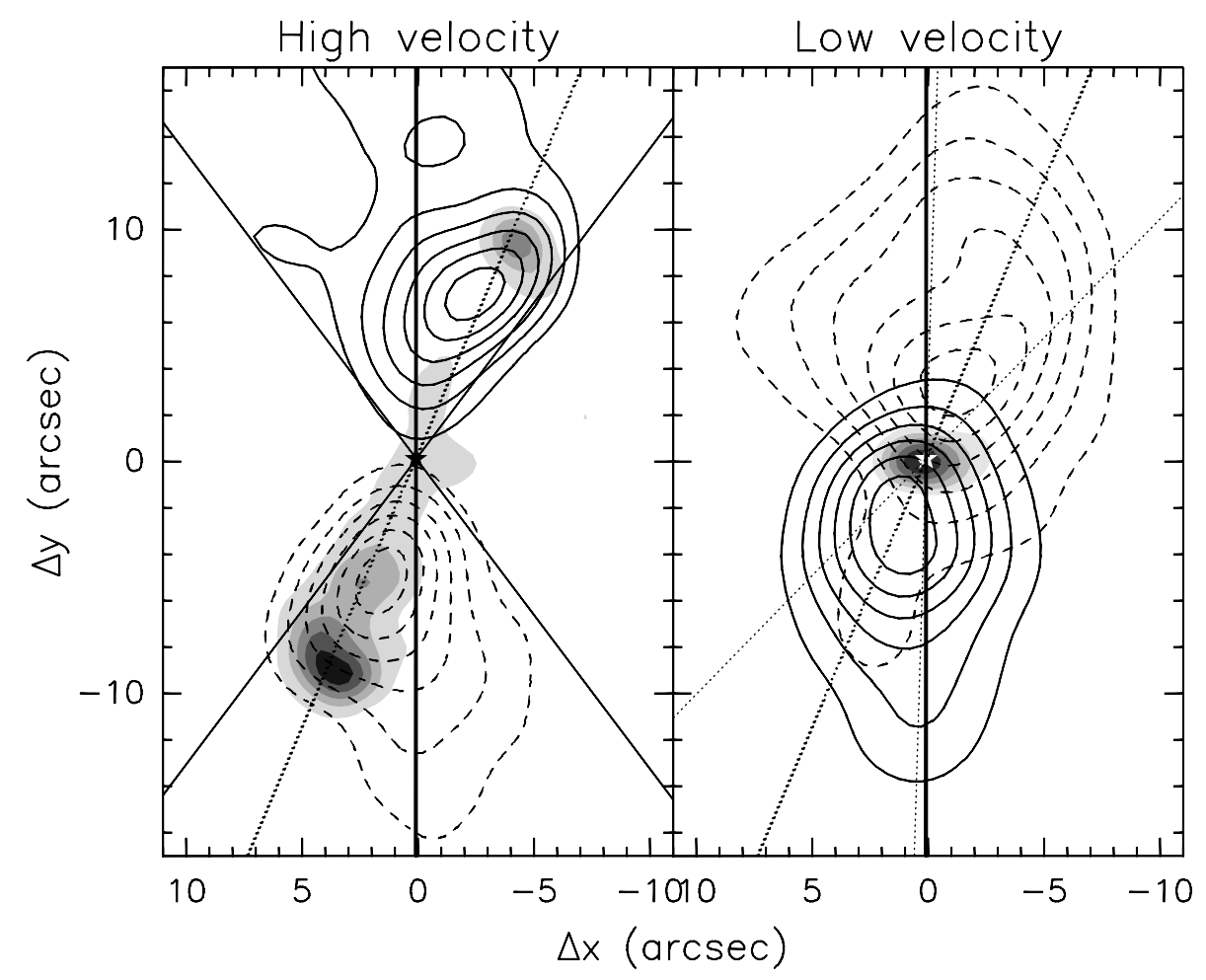

Fig. 17. Maps of the $\mathrm{HCO}^{+}$outflow (from C97) after rotation by $37^{\circ}$. The low-velocity flow has been obtained integrating the line emission from -6.9 to $-5.6 \mathrm{~km} \mathrm{~s}^{-1}$ (full contours) and from -2.4 to $-1.4 \mathrm{~km} \mathrm{~s}^{-1}$ (dashed), whereas for the high-velocity maps the integration was done from -28.2 to $-16.3 \mathrm{~km} \mathrm{~s}^{-1}$ (full contours) and from 5.2 to $19.9 \mathrm{~km} \mathrm{~s}^{-1}$ (dashed). Contour levels range from $15 \%$ to $90 \%$ by $15 \%$ of the corresponding peak intensity. Also shown are grey scale maps of the disk in the integrated $\mathrm{C}^{34} \mathrm{~S}(2-1)$ line emission (right panel) and $\mathrm{SiO}(2-1)$ outflow $($ left panel) from C99. The thick and thin solid lines indicate respectively the disk rotation axis and the projection of the precession cone onto the plane of the sky. The thick and thin dotted lines indicate respectively the current position of the outflow axis and the projection onto the plane of the sky of the outflow cone. The star marks the position of the $1.3 \mathrm{~mm}$ continuum peak.

\subsubsection{A model for the precessing outflow}

The best tracers to identify this phenomenon are the $\mathrm{H}_{2}$ and $\mathrm{H} \alpha$ knots detected by C99 and SYBT over a region as large as $\sim 2^{\prime}$ $(\sim 1 \mathrm{pc})$. These show the projection onto the plane of the sky of the trajectory drawn by a bullet shot along the precessing axis of the flow. Therefore, the knots must lie over the surface of the cone described by the outflow axis during its precession. In our model we assume that the knots have radial velocities diverging from the vertex of the cone and that the precession occurs with constant angular velocity about the rotation axis of the disk. Under these hypotheses, the model is described by five parameters: the RA and Dec of the vertex, the inclination angle $(\psi)$ between the disk axis and the plane of the sky, the opening angle $(\theta)$ of the precession cone, and the ejection velocity $(\dot{R})$, which may be expressed as a function of the distance $R$ from the vertex.

The coordinates of the vertex are given by the peak of the millimeter continuum, which is a good approximation to the position of the embedded YSO powering the outflow.

An estimate of $\theta$ is given by the maximum angle between the disk axis and the outflow axis: since the position angle of the former is $-37^{\circ}$ and the oldest direction of the outflow axis corresponds to the $\mathrm{N}-\mathrm{S}$ orientation of the ${ }^{12} \mathrm{CO}$ flow observed by SYBT, very likely $\theta \simeq 37^{\circ}$.
It is unlikely that the disk axis is significantly inclined with respect to the plane of the sky. In fact, if $\psi=0$ the outflow axis will lie in the plane of the sky when its angle with the disk axis will be equal to the opening angle of the precession cone, i.e. $37^{\circ}$. Indeed, $\mathrm{C} 99$ have demonstrated that the current direction of the outflow axis lies very close $\left(\sim 9^{\circ}\right)$ to the plane of the sky and that the projection of such axis onto the plane of the sky forms an angle of $\sim 23^{\circ}$ with the disk axis, i.e. close to $\theta=37^{\circ}$. We may hence assume $\psi=0$ in the following.

With this in mind, one can estimate the expansion velocity law of the flow. Since the precession angular velocity $(\dot{\phi})$ is constant, the precession angle $(\phi)$ can be taken as a measure of time. Moreover, the 3D geometry of the precession cone is known, so that it is possible to compute $\phi$ and $R$, for each knot using the following expressions:

$$
\begin{aligned}
\tan ^{2} \phi & =\frac{y^{2} \tan ^{2} \theta-x^{2}}{x^{2}} \\
R^{2} & =\frac{y^{2}}{\cos ^{2} \theta}
\end{aligned}
$$

under the assumption of $\psi=0$ and with $x, y$ reference system centred on the star and lying in the plane of the sky, with $y$ representing the projection of the disk axis. A plot of $R$ versus $\phi$ for the knots in Table 1 of SYBT is shown in the upper panel of Fig. 18. The two quantities are reasonably correlated according 

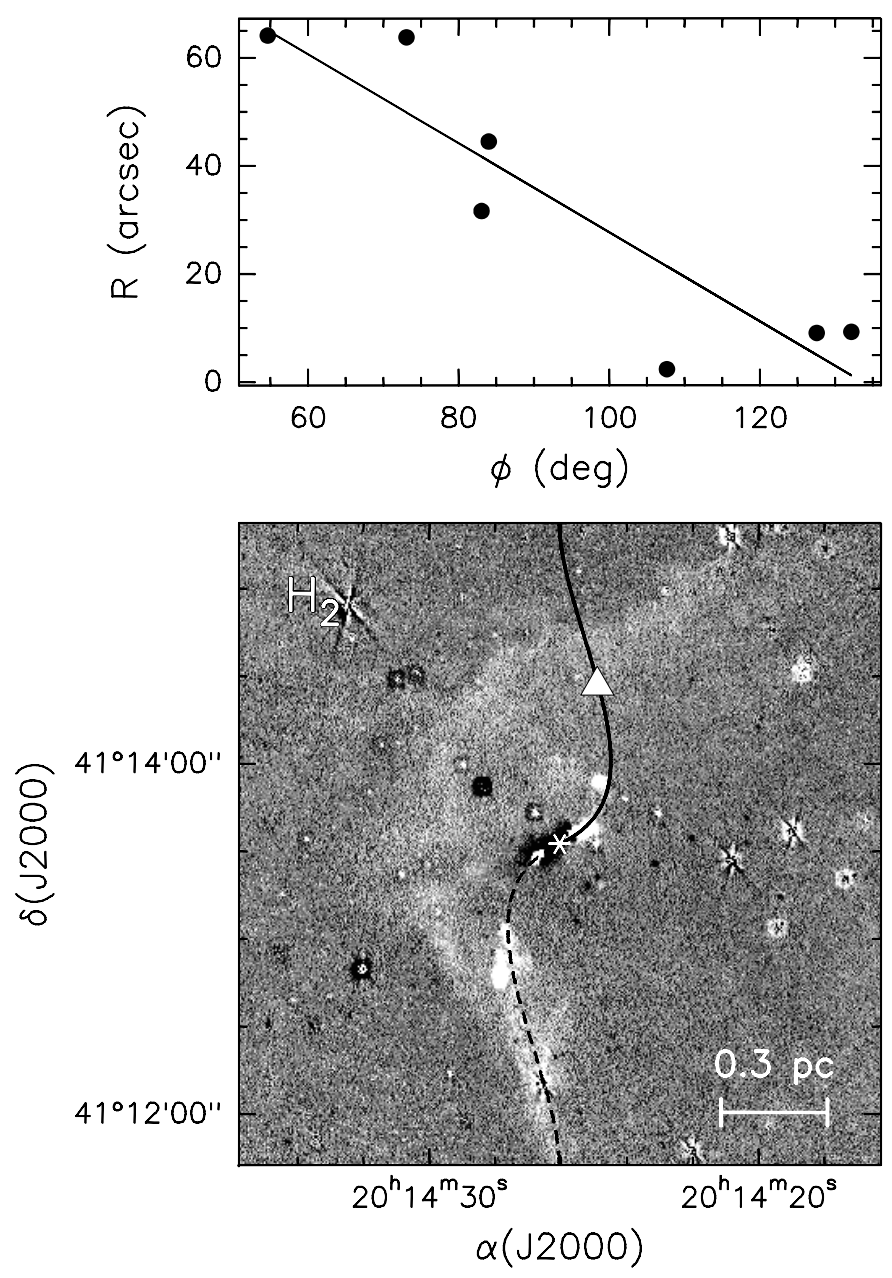

Fig. 18. Upper panel: plot of the distance $R$ from the centre of the outflow versus the angle $\phi$ between the projection of the precessing outflow axis onto the disk plane and the intersection between the disk plane and the plane of the sky. The points represent the $\mathrm{H}_{2}$ and $\mathrm{H} \alpha$ knots listed in Table 1 of SYBT. The straight line is a least square fit to the data. Lower panel: image of the $\mathrm{H}_{2}$ emission with overlaied the trajectory described in the plane of the sky by a bullet shot along the flow axis assuming $\dot{\phi}=$ const. and the linear dependence between $R$ and $\phi$ represented by the least square fit in the upper panel. The full and dashed parts of the curve correspond respectively to blue- and red-shifted velocities. The triangle marks the position of the $\mathrm{H} \alpha$ knot.

to the law $R(\operatorname{arcsec})=-0.69 \phi(\mathrm{deg})+97$ : this result is consistent with a constant expansion velocity, as $\phi$ depends linearly on time. Note that by imposing $R=0$ one obtains the current direction of the outflow axis, which is $\phi=134^{\circ}$.

Using the previous values of $\psi, \theta$, and $R(\phi)$, one can compute the trajectory of a bullet emitted by the precessing flow: this is drawn in the lower panel of Fig. 18 over our new TNG $\mathrm{H}_{2}$ image of the region. Clearly, the agreement with the $\mathrm{H}_{2}$ and $\mathrm{H} \alpha$ knots is excellent, thus confirming the validity of the model. Finally, from the relationship between $R$ and $\phi$ it is easy to compute the angular velocity of the precession, assuming the expansion velocity of $100 \mathrm{~km} \mathrm{~s}^{-1}$ estimated by C99: $\dot{\phi} \simeq 0.018 \mathrm{deg} \mathrm{yr}^{-1}$. The lifetime of the flow is greater or equal to the dynamical time of the outflow (64000 yr) estimated by SYBT (see their Table 2): this implies that to date the flow has precessed by at least $1150^{\circ}$. It is worth noting that the outflow age is 10 times greater than the time required by the farthest knot to reach the current position expanding at a speed of $100 \mathrm{~km} \mathrm{~s}^{-1}$ : this suggests that more knots might be found on a larger scale if images with sufficient field of view and sensitivity will be available.

The origin of the precession has been extensively discussed by SYBT and will not be further investigated here. These authors concluded that the precession could be caused by the interaction between the disk and a stellar companion orbiting about the (proto)star at the disk centre. One may speculate that such a companion might be located at the position of the secondary peak seen in the $\mathrm{CH}_{3} \mathrm{CN}(12-11) K=8$ line (see Sect. 4.1).

\subsubsection{The S-shaped low-velocity feature}

An important consequence of the fit obtained in the previous section is that at present the outflow axis is about to cross the plane of the sky, with the NW part of it receding from the observer and the SE part approaching him. Since the precession is slow compared to the ejection, the result is that at any given time the material surrounding the outflow lobes is not homogeneously distributed around them: the region left behind in the precession has been swept away by the flow itself, whereas the molecular gas towards which the flow is precessing is still unaffected.

For example, let us consider the NW lobe of the outflow. The blue-shifted, high-velocity emission (see left panel of Fig. 17) is contributed by the part of this lobe that still lies in the halfspace of the observer. Figure 17 illustrates that the $\mathrm{SiO}$ flow is aligned along the current direction of the outflow axis (dotted line), while the bow shocks at the head of it mark the place where the ejected material is impinging against the surrounding molecular cloud. The lobe is precessing around the disk axis (thick vertical line) moving from left to right, thus approacing the intersection of the precession cone with the plane of the sky (full line in the left panel of Fig. 17). It is interesting to note that such a precession is revealed by the wake of emission left behind the outflow lobes.

On the other hand, the part of the NW lobe that has crossed the plane of the sky contributes to the red-shifted, low-velocity emission (right panel of Fig. 17), mainly for two reasons: it is moving almost perpendicularly to the line of sight and it is entraining fresh molecular gas which increases the density of the flow, enriches its composition, and brakes the expansion. Consequently, the red-shifted line intensity is boosted by the density increase and molecular enrichment unlike the blueshifted gas which, lying closer to the evacuated region, is much less dense and subject to higher expansion velocities.

The previous scenario explains the S-shaped asymmetry in the position velocity plots. Also, it solves the paradox that most molecules seem to trace blue-shifted emission to the NW and red-shifted to the SE, while we know that this should not be the case since the outflow axis points towards the observer in the NW part. The explanation is that molecular abundances are much more prominent in front of the precessing lobes. 


\subsection{Infall and nature of the YSO}

What is the nature of the YSO in IRAS 20126+4104? The question we want to address here is whether one is dealing with a ZAMS star or with a protostar. For this purpose it is helpful to compare the luminosity and the mass of the star. The latter has been evaluated in Sect. 4.1.3 as $\sim 7 M_{\odot}$, while the former is $\sim 10^{4} L_{\odot}$ (C99). The luminosity of a ZAMS with a similar mass should be $\sim 10^{3} L_{\odot}$, an order of magnitude less than that of IRAS 20126+4104: such a discrepancy may be explained if IRAS 20126+4104 is a protostar deriving its luminosity from accretion. In fact, according to the computation of Behrend \& Maeder (2001), $\sim 10^{4} L_{\odot}$ is just the total luminosity of a $\sim 7 M_{\odot}$ protostar plus disk (see their Table 1). In their model, an accretion rate of $10^{-3} M_{\odot} \mathrm{yr}^{-1}$ is required: is this consistent with our observations?

As noted in Sect. 3, in the $\mathrm{C}^{34} \mathrm{~S}$ spectra one may see a hint of red-shifted self-absorption, suggestive of infall in the disk. As a matter of fact, the presence of infall in massive star forming regions has been observationally established (see e.g. Keto et al. 1988; Ho et al. 1996; Sollins et al. 2004) and theoretically predicted (Keto 2003) as a crucial phenomenon profoundly affecting the formation of early-type stars. In order to estimate the accretion rate, we use the same approach as in C99:

$\dot{M}_{\text {acc }}=2 \pi R \frac{M(R)}{4 \pi R^{2}} \sqrt{\frac{2 G M_{*}}{R}}$

where $M(R)$ is the mass of the disk inside a given radius $R$. From Table 3 one sees that the mass of the disk $\left(\sim 4 M_{\odot}\right)$ traced by our millimeter continuum observations is enclosed inside a radius of $760 \mathrm{AU}$. Substituting these values in the previous equation one obtains $\dot{M}_{\text {acc }} \simeq 2 \times 10^{-3} M_{\odot} \mathrm{yr}^{-1}$, which agrees well with the value derived above from the luminosity of the source. Such an accretion rate is bound to exhaust the material in the disk after about $4 M_{\odot} / 2 \times 10^{-3} M_{\odot} \mathrm{yr}^{-1}=2 \times 10^{3} \mathrm{yr}$, a short time compared to the outflow age quoted by SYBT $\left(6.4 \times 10^{4} \mathrm{yr}\right)$. Since it is reasonable that the disk and outflow are physically related entities, their lifetimes are likely similar to each other: this implies that the disk must be continuously fed through accretion from the surrounding parsec-scale envelope. It must be noted that the accretion rate estimated above is computed under the assumption that all the infalling material will accrete onto the star, whereas theoretical models predict that a fraction of it will contribute to the outflow. However, this fraction is expected to be relatively small, $\sim 30-50 \%$ (see Behrend \& Maeder 2001 and references therein). As a matter of fact, the outflow rate estimated by SYBT is $8.1 \times$ $10^{-4} M_{\odot} \mathrm{yr}^{-1}$, i.e. $40 \%$ of $\dot{M}_{\text {acc }}$. We hence conclude that $2 \times 10^{-3} M_{\odot} \mathrm{yr}^{-1}$ is very close to the accretion rate onto the star.

\section{Summary and conclusions}

We have performed observations with the Plateau de Bure interferometer towards the high-mass young stellar object IRAS 20126+4104. New images in the $\mathrm{H}_{2}$ line obtained with the Telescopio Nazionale Galileo are also presented. Our findings confirm the existence of a Keplerian disk and bipolar outflow oriented roughly perpendicularly to the disk. The outflow axis turns out to be precessing as described by SYBT. A simple model is elaborated to describe such a precession and thus derive the corresponding angular velocity $\left(0.018{\mathrm{deg} \mathrm{r}^{-1}}^{-1}\right.$.

The mass of the central YSO is obtained by fitting a numerical model to the position velocity plots of the $\mathrm{C}^{34} \mathrm{~S}$ lines. This turns out to be $\sim 7 M_{\odot}$, namely $2-3$ times less than the estimates obtained with the same method from the $\mathrm{NH}_{3}(1,1)$ (Zhang et al. 1998) and $\mathrm{HCO}^{+}$lines. We believe that such a discrepancy may be explained by the fact that the disk mass enclosed in the region traced by the $\mathrm{NH}_{3} \mathrm{HCO}^{+}$is non negligible with respect to the mass of the YSO.

Line ratios of the $\mathrm{C}^{34} \mathrm{~S}$ and $\mathrm{CH}_{3} \mathrm{OH}$ lines prove the existence of a temperature and density gradient in the disk, while the line profiles indicate that the material of the disk might be infalling onto the central YSO at a rate of $\sim 2 \times 10^{-3} M_{\odot} \mathrm{yr}^{-1}$. According to current theoretical models (Behrend \& Maeder $2001)$, this is just the accretion rate expected for a $7 M_{\odot}$ protostar with the luminosity of IRAS $20126+4104\left(\sim 10^{4} L_{\odot}\right)$. We hence conclude that IRAS $20126+4104$ is likely a massive protostar in the accretion phase.

Acknowledgements. It is a pleasure to thank the staff of IRAM for their help during the observations. $\mathrm{PH}$ acknowledges partial support from Research Corporation award CC4996 and from NSF grant AST0098524. This paper is partly based on observations made with the Italian Telescopio Nazionale Galileo (TNG) operated on the island of La Palma by the Centro Galileo Galilei of the INAF (Istituto Nazionale di Astrofisica) at the Spanish Observatorio del Roque de los Muchachos of the Instituto de Astrofisica de Canarias. The ARNICA/TNG observations were performed in service mode by the TNG staff, we especially acknowledge the help of Francesca Ghinassi and Juan Carlos Guerra. Many thanks are also due to the referee, Paul Ho, for careful reading of the manuscript and helpful comments.

\section{Appendix A: Mean kinetic temperature in a Keplerian disk}

In this appendix, we elaborate a simplified analytical expression for the mean kinetic temperature $(T)$ in the plane of the sky for the material moving in a Keplerian disk, at a given velocity $(v)$ along the line of sight. In the following we assume that all quantities are normalised with respect to the outer radius of the disk, $R_{0}$, and the rotation velocity at such radius, $v_{0}$ : therefore in our notation $x$ stands for $x / R_{0}, R$ (the distance from the centre of the disk) for $R / R_{0}$, and $v$ for $v / v_{0}$. Moreover, since the mean value of $T$ must be positive, independently of the sign of $x$ and $v$, in the following we shall replace these quantities by their absolute values, i.e. $x$ will stand for $|x|$ and $v$ for $|v|$.

Figure A.1 shows the outer border of the disk (corresponding to $R=1$ ) and the curves corresponding to points with velocity $v$ for two cases: $v>1$ (curve to the left) and $v<1$ (curve to the right). It is convenient to express such curves as

$x=v R^{\frac{3}{2}}$

The mean value of $T$, measured in the plane of the sky over the whole disk, is obtained integrating the temperature along the 


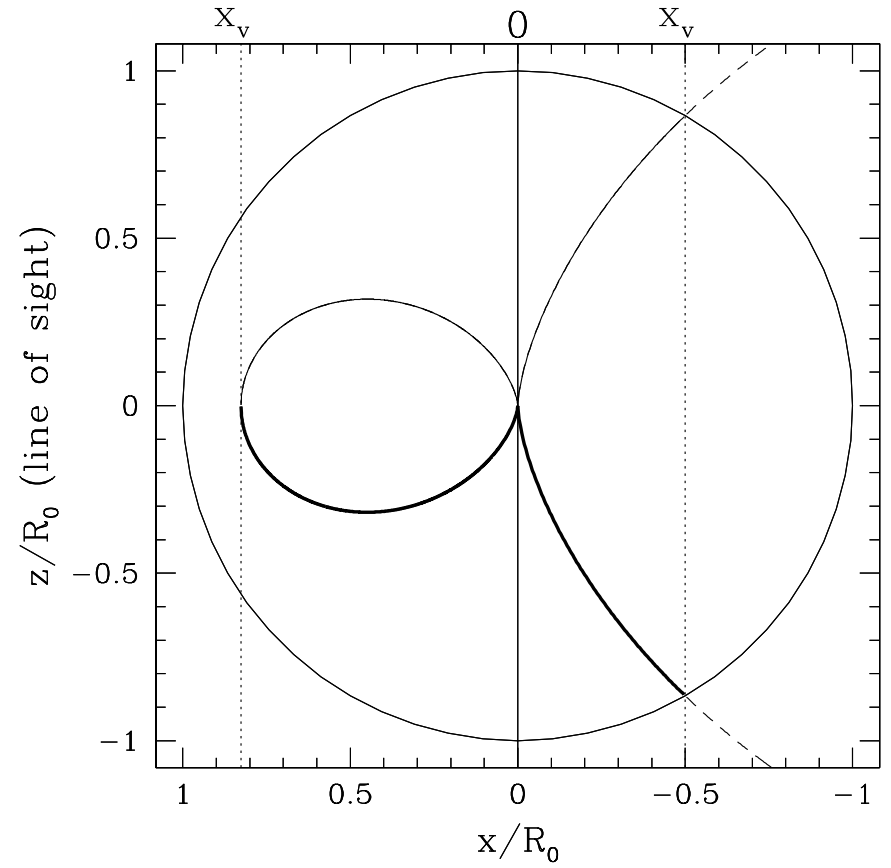

Fig. A.1. Loci of points in a Keplerian disk corresponding to a given velocity $v$ along the line of sight. All quantities are normalised with respect to the outer radius of the disk and the corresponding rotation velocity. The curve to the right is for $|v|<1$ and the one to the left for $|v|>1$. The thick portion of each curve indicates the pattern over which the integral in Eq. (A.2) must be performed. The maximum value of $x$ attainable for each curve inside the disk $\left(x_{v}\right)$ is indicated by the dotted lines. The line of sight is parallel to the $z$-axis.

thick portion of the curves in Fig. A.1 and is hence given by the expression

$\langle T\rangle_{v}=\frac{\int_{0}^{x_{v}} T \mathrm{~d} x}{x_{v}}$

where $x_{v}$ is the maximum value of $x$ for a given curve. This is attained for $R=1$, if $v \leq 1$, and for $R=x$, if $v \geq 1$, so that it may be expressed as

$x_{v}=\min \left\{v, v^{-2}\right\}$.

We assume that $T$ depends on the radius as a power law, hence using Eq. (A.1) one can write

$T=T_{0}\left(\frac{x}{v}\right)^{\frac{2}{3}}$
Substituting Eqs. (A.3) and (A.4) in Eq. (A.2), one can evaluate the integral and thus obtain Eq. (1):

$\langle T\rangle_{v}=\frac{T_{0}}{\frac{2}{3} q+1}\left(\min \left\{1, v^{-3}\right\}\right)^{\frac{2}{3} q}$.

\section{References}

Baffa, C., Comoretto, G., Gennari, S., et al. 2001, A\&A, 378, 722

Behrend, R., \& Maeder, A. 2001, A\&A, 373, 190

Bertin, G., \& Lodato, G. 1999, A\&A, 350, 694

Cesaroni, R., Walmsley, C. M., Kömpe, C., \& Churchwell, E. 1991, A\&A, 252, 278

Cesaroni, R., Felli, M., Testi, L., Walmsley, C. M., \& Olmi, L. 1997, A\&A, 325, 725 (C97)

Cesaroni, R., Felli, M., Jenness, T., et al. 1999a, A\&A, 345, 949 (C99)

Cesaroni, R., Felli, M., \& Walmsley, C. M. 1999b, A\&AS, 136, 333

Comoretto, G., Palagi, F., Cesaroni, R., et al. 1990, A\&AS, 84, 179

Estalella, R., Mauersberger, R., Torrelles, J. M., et al. 1993, ApJ, 419, 698

Ghinassi, F., Licandro, J., Oliva, E., et al. 2002, A\&A, 386, 1157

Guilloteau, S., Delannoy, J., Downes, D., et al. 1992, A\&A, 262, 624

Hildebrand, R. H. 1983, QJRAS, 24, 267

Ho, P. T. P., \& Young, L. M. 1996, ApJ, 472, 742

Hofner, P., Cesaroni, R., Rodríguez, L. F., \& Martí, J. 1999, A\&A, 345, L43

Kawamura, J. H., Hunter, T. R., Tong, C.-Y. E., et al. 1999, PASP, 111, 1088

Keto, E. H. 2003, ApJ, 599, 1196

Keto, E. H., Ho, P. T. P., \& Haschick, A. D. 1988, ApJ, 324, 920

Kramer, C., Richer, J., Mookerjea, B., Alves, J., \& Lada, C. 2003, A\&A, 399, 1073

Kurtz, S., Hofner, P., \& Vargas Alvarez, C. 2004, ApJS, 155, 149

Moscadelli, L., Cesaroni, R., \& Rioja, M. J. 2000, A\&A, 360, 663

Ossenkopf, V., \& Henning, Th. 1994, A\&A, 291, 943

Palla, F., \& Stahler, S. W. 1993, ApJ, 418, 414

Pringle, J. E. 1981, ARA\&A, 19, 137

Simon, M., Guilloteau, S., \& Dutrey, A. 2001, ApJ, 545, 1034

Shepherd, D. S., Yu, K. C., Bally, J., \& Testi, L. 2000, ApJ, 535, 833 (SYBT)

Sollins, P. K., Zhang, Q., \& Ho, P. T. P. 2004, ApJ, 606, 943

Tofani, G., Felli, M., Taylor, G. B., \& Hunter, T. R. 1995, A\&AS, 112, 299

Van Dishoeck, E. F., Blake, G. A., Draine, B. T., \& Lunine, J. I. 1993, Protostars and Planets III, ed. E. H. Levy, \& J. I. Lunine (Tucson: Univ. of Arizona Press)

Wilking, B. A., Blackwell, J. H., \& Mundy, L. G. 1990, AJ, 100, 758

Wood, D. O. S., \& Churchwell, E. 1989, ApJ, 340, 265

Zhang, Q., Hunter, T. R., \& Sridharan, T. K. 1998, ApJ, 505, L151

Zhang, Q., Hunter, T. R., Sridharan, T. K., \& Cesaroni, R. 1999, ApJ, 527, L117 